\title{
Mean-field theory of Bayesian clustering
}

\author{
Alexander Mozeika ${ }^{1}$ and Anthony CC Coolen ${ }^{1,2}$ \\ ${ }^{1}$ Institute for Mathematical and Molecular Biomedicine, \\ King's College London, Hodgkin Building, London SE1 1UL, UK. \\ ${ }^{2}$ Department of Mathematics, King's College London, \\ The Strand, London WC2R 2LS, UK.
}

(Dated: August 4, 2021)

\begin{abstract}
We show that model-based Bayesian clustering, the probabilistically most systematic approach to the partitioning of data, can be mapped into a statistical physics problem for a gas of particles, and as a result becomes amenable to a detailed quantitative analysis. A central role in the resulting statistical physics framework is played by an entropy function. We demonstrate that there is a relevant parameter regime where mean-field analysis of this function is exact, and that, under natural assumptions, the lowest entropy state of the hypothetical gas corresponds to the optimal clustering of data. The byproduct of our analysis is a simple but effective clustering algorithm, which infers both the most plausible number of clusters in the data and the corresponding partitions. Describing Bayesian clustering in statistical mechanical terms is found to be natural and surprisingly effective.

PACS numbers: $02.50 . \mathrm{Tt}$, 05.10.-a, 64.60.De
\end{abstract}




\section{INTRODUCTION}

The need for clustering analysis in scientific data exploration has grown significantly in recent years, due to the emergence of large high-dimensional datasets in areas such as high energy physics, astrophysics, biology and post-genome medicine. The aim of clustering analysis is to allocate similar data items, such as stars [1], galaxies [2], bacterial communities [3], or amino-acid sequences [4], to the same category (or 'cluster') in an unsupervised way. Inferring the true number of clusters reliably is crucial for the discovery of new data categories. Most current clustering methods, such as [5 7], make no assumptions about the data distribution, and are based on heuristic measures of similarity. Some allow for estimation of the number of clusters, but use empirical approaches to do so and ad-hoc evaluation criteria tested on benchmark datasets.

Model-based clustering assumes that each data point comes from one of a postulated number of populations with known distributions. The archetypal example is the Gaussian Mixture Model (GMM) [5], which assumes Gaussian distributions. In such models Maximum likelihood (ML) inference is typically used to find data partitions [8], but this is prone to overfitting [5]. The number of clusters $K$ is found upon adding a 'penalty' term to the loglikelihood function, such as AIC or BIC [8], sometimes with conflicting results [2]. Bayesian inference of GMM-generated data cures overfitting and provides a systematic way to find $K$ [5]. However, computing the posteriors is analytically intractable, and one tends to resort to either variational mean-field approximation [5] or computationally intensive MCMC [9].

A more general model-based Bayesian clustering protocol (SPD) was introduced in [10]. Unlike GMM, it uses priors on the partitions to compute a maximum a posteriori probability (MAP) estimate of the data partitioning. Both SPD and GMM Bayesian methods are usually evaluated by clustering synthetic and benchmark real-world data. This is not satisfactory; one would prefer our knowledge and our confidence in clustering outcomes to be based on more than empirical tests.

As a first step in this direction, in this paper we use statistical physics to study modelbased Bayesian clustering. This strategy was used in the past to study optimization problems, see e.g. [11], and clustering [12, 13], but not Bayesian clustering. Starting from the SPD model, we show that data partition inference can be formulated in terms of a quantity that can be seen as the entropy of a gas of a particles (data-points), distributed over $K$ 
reservoirs (clusters). In the regime of a large number of particles we derive a mean-field theory to describe this gas, and show that its lowest entropy state corresponds to the optimal MAP clustering of data.

\section{MODEL OF DATA AND BAYESIAN CLUSTERING}

Let us assume that we observe the sample $\mathbf{X}=\left\{\mathbf{x}_{1}, \ldots, \mathbf{x}_{N}\right\}$, with $\mathbf{x}_{i} \in \mathbb{R}^{d}$ for all $i$, from the distribution

$$
p(\mathbf{X} \mid \boldsymbol{\Theta}, \Pi)=\prod_{\mu=1}^{|\Pi|} \prod_{i_{\mu} \in S_{\mu}} p\left(\mathbf{x}_{i_{\mu}} \mid \boldsymbol{\theta}_{\mu}\right) .
$$

This distribution is generated by the set (or 'partition') $\Pi=\left\{S_{1}, \ldots, S_{|\Pi|}\right\}$, with disjunct index sets (or 'clusters') $S_{\mu} \neq \emptyset$, such that $S_{\mu} \cap S_{\nu}=\emptyset$ for $\mu \neq \nu$ and $\cup_{\mu=1}^{|\Pi|} S_{\mu}=[N]$ with $[N]=\{1, \ldots, N\}$. Any partition of data into $K$ clusters can be specified by binary 'cluster allocation' variables $c_{i \mu}=\mathbb{1}\left[i \in S_{\mu}\right]$, where $i \in[N]$ and $\mu \in[K]$, forming an $N \times K$ partitioning

matrix c. This matrix satisfies by construction the following constraints: $\sum_{\mu=1}^{K} c_{i \mu}=1$ for all $i \in[N]$, and $\sum_{i=1}^{N} c_{i \mu} \geq 1$ for all $\mu \in[K]$. Conversely, any $N \times K$ matrix $\mathbf{c} \in\{0,1\}^{N K}$ with binary entries that satisfies these constraints induces a partition $\Pi(\mathbf{c})=\left\{S_{1}(\mathbf{c}), \ldots, S_{K}(\mathbf{c})\right\}$ of cardinality $K$. If we also know the prior distributions of model parameters, $p\left(\boldsymbol{\theta}_{\mu}\right), p(\mathbf{c} \mid K)$ and $p(K)$, we can use Bayes' theorem (see Appendix $\mathrm{A}$ for details) to derive the posterior distribution

$$
p(\mathbf{c}, K \mid \mathbf{X})=\frac{\mathrm{e}^{-N \hat{F}_{N}(\mathbf{c}, \mathbf{X})} p(\mathbf{c} \mid K) p(K)}{\sum_{\tilde{K}=1}^{N} p(\tilde{K}) \sum_{\tilde{\mathbf{c}}} \mathrm{e}^{-N \hat{F}_{N}(\tilde{\mathbf{c}}, \mathbf{X})} p(\tilde{\mathbf{c}} \mid \tilde{K})},
$$

in which

$$
\hat{F}_{N}(\mathbf{c}, \mathbf{X})=-\frac{1}{N} \log \left\langle\mathrm{e}^{\sum_{\mu=1}^{K} \sum_{i=1}^{N} c_{i \mu} \log p\left(\mathbf{x}_{i} \mid \boldsymbol{\theta}_{\mu}\right)}\right\rangle_{\boldsymbol{\Theta}}
$$

with $\langle f(\boldsymbol{\Theta})\rangle_{\boldsymbol{\Theta}}=\int\left[\prod_{\mu=1}^{K} p\left(\boldsymbol{\theta}_{\mu}\right) \mathrm{d} \boldsymbol{\theta}_{\mu}\right] f(\boldsymbol{\Theta})$. Expression (2) can be used to infer the most probable partition $\Pi$ for each data sample. First, for each $K \in[N]$ one computes

$$
\hat{\mathbf{c}} \mid K=\operatorname{argmax}_{\mathbf{c}}\left\{\mathrm{e}^{-N \hat{F}_{N}(\mathbf{c}, \mathbf{X})} p(\mathbf{c} \mid K)\right\} .
$$

Then one uses (44) to determine the estimate $\hat{\Pi}$ of $\Pi$ :

$$
\hat{\Pi}=\operatorname{argmax}_{\hat{\mathbf{c}} \mid K}\left\{\mathrm{e}^{-N \hat{F}_{N}(\hat{\mathbf{c}}, \mathbf{X})} p(\hat{\mathbf{c}} \mid K) p(K)\right\} .
$$


Clearly, a key role in our formulae is played by the function (3), which can be seen as an entropy of a gas of $N$ 'particles' (the data-points) distributed over $K$ 'reservoirs' (clusters). The particles can move from one reservoir to another; $c_{i \mu}$ tells us if particle $i$ is in reservoir $\mu$, and the coordinates $\mathbf{x}_{i}$ act as a 'quenched' disorder [14]. We are then interested in the minimum entropy state $\operatorname{argmin}_{\mathbf{c}} \hat{F}_{N}(\mathbf{c}, \mathbf{X})$.

\section{MEAN-FIELD ANALYSIS OF BAYESIAN CLUSTERING}

Let us first consider the case where the cluster parameters are known. In this case the parameter prior $p\left(\boldsymbol{\theta}_{\mu}\right)$ is a delta function, and (3יi) hence becomes

$$
\hat{F}_{N}(\mathbf{c}, \mathbf{X})=-\sum_{\mu=1}^{K} \frac{M_{\mu}(\mathbf{c})}{N} \int \mathrm{d} \mathbf{x} \hat{Q}_{\mu}(\mathbf{x} \mid \mathbf{c}, \mathbf{X}) \log p\left(\mathbf{x} \mid \boldsymbol{\theta}_{\mu}\right),
$$

which is now written in terms of the number of particles in cluster $\mu, M_{\mu}(\mathbf{c})=\sum_{i=1}^{N} c_{i \mu}$, and the density of particles in cluster $\mu$, defined as

$$
\hat{Q}_{\mu}(\mathbf{x} \mid \mathbf{c}, \mathbf{X})=\frac{1}{M_{\mu}(\mathbf{c})} \sum_{i=1}^{N} c_{i \mu} \delta\left(\mathbf{x}-\mathbf{x}_{i}\right) .
$$

Suppose there are $L$ distributions $q_{\nu}(\mathbf{x})$, such that for each $\nu$ we find $N_{\nu}$ particles with $\mathbf{x}_{i}$ sampled from $q_{\nu}(\mathbf{x})$, with $\sum_{\nu=1}^{L} N_{\nu}=N$ and $\lim _{N \rightarrow \infty} N_{\nu} / N=\gamma(\nu)$. For large $N$ the density (7) will then typically converge to

$$
Q_{\mu}(\mathbf{x})=\sum_{\nu=1}^{L} \alpha(\nu \mid \mu) q_{\nu}(\mathbf{x})
$$

Here $\alpha(\nu \mid \mu)=\alpha(\nu, \mu) / \alpha(\mu)$ is a conditional probability, defined by $\alpha(\mu)=\lim _{N \rightarrow \infty} M_{\mu}(\mathbf{c}) / N$ and $\alpha(\nu, \mu)=\lim _{N \rightarrow \infty} M_{\nu, \mu}(\mathbf{c}) / N$, where $M_{\mu}(\mathbf{c})$ is the number of particles in cluster $\mu$ and $M_{\nu, \mu}(\mathbf{c})=\sum_{i_{\nu \in S_{\mu}(\mathbf{c})}} \mathbb{1}\left[\mathbf{x}_{i_{\nu}} \sim q_{\nu}(\mathbf{x})\right]$ is the number of those particles drawn from the distribution $q_{\nu}(\mathbf{x})$ that are allocated by $\mathbf{c}$ to cluster $\mu$. Clearly $\sum_{\mu \leq K} \alpha(\nu, \mu)=\gamma(\nu), \sum_{\nu \leq L} \alpha(\nu, \mu)=$ $\alpha(\mu)>0$ and $\sum_{\nu \leq L} \sum_{\mu \leq K} \alpha(\nu, \mu)=1$. If (8) holds for $N \rightarrow \infty$, then $\hat{F}_{N}(\mathbf{c}, \mathbf{X})$ will for $N \rightarrow \infty$ converge to

$$
F(\boldsymbol{\alpha})=\sum_{\mu=1}^{K} \sum_{\nu=1}^{L} \alpha(\nu, \mu) D\left(q_{\nu}|| p_{\mu}\right)+\sum_{\nu=1}^{L} \gamma(\nu) H\left(q_{\nu}\right) .
$$

Here $D\left(q_{\nu} \| p_{\mu}\right)$ is the Kullback-Leibler distance between $q_{\nu}(\mathbf{x})$ and $p\left(\mathbf{x} \mid \boldsymbol{\theta}_{\mu}\right)$, and $H\left(q_{\nu}\right)$ is a differential entropy [15]. The transparent and intuitive result (9) can be seen as a mean-field 
$(\mathrm{MF})$ theory of $\hat{F}_{N}(\mathbf{c}, \mathbf{X})$ (see Appendix $\mathrm{C}$ for details). The $L \times K$ matrix $\boldsymbol{\alpha}$, with entries $\alpha(\nu, \mu)$, acts as order parameter. More generally one would have $P(F)=\int \mathrm{d} \boldsymbol{\alpha} P(\boldsymbol{\alpha}) \delta(F-$ $F(\boldsymbol{\alpha}))$, where

$$
P(\boldsymbol{\alpha})=\lim _{N \rightarrow \infty} \sum_{\mathbf{c}, \tilde{\mathbf{c}}} p(\mathbf{c} \mid K) q(\tilde{\mathbf{c}} \mid L) \prod_{\mu=1}^{K} \prod_{\nu=1}^{L} \delta\left[\alpha(\nu, \mu)-\frac{1}{N} \sum_{i=1}^{N} \tilde{c}_{i \nu} c_{i \mu}\right] .
$$

Here $p(\mathbf{c} \mid K)$ and $q(\tilde{\mathbf{c}} \mid L)$ are the assumed and the 'true' distributions of partitions, respectively. We can limit ourselves to working with expression (9), as opposed to the more involved (10), if $P(\boldsymbol{\alpha})$ is a delta function.

We are interested in the state $\boldsymbol{\alpha}$ for which the function $F(\boldsymbol{\alpha})$ is minimal. Firstly, from $D\left(q_{\nu} \| p_{\mu}\right) \geq 0$ it follows that $F(\boldsymbol{\alpha}) \geq \sum_{\nu=1}^{L} \gamma(\nu) H\left(q_{\nu}\right)$. The lower bound is saturated when $D\left(q_{\nu}|| p_{\mu}\right)=0$, i.e. when $q_{\nu}(\mathbf{x})=p\left(\mathbf{x} \mid \boldsymbol{\theta}_{\mu}\right)$ for all $(\mu, \nu)$, and the mapping between the sets $[L]$ and $[K]$ labelling these distributions is bijective. This can only happen when $L=K$ and $\alpha(\nu, \mu)=\gamma(\nu) \mathbb{1}\left[D\left(q_{\nu}|| p_{\mu}\right)=0\right]$, i.e. when the 'true' partitioning of the data is recovered.

Secondly, from $D\left(q_{\nu}|| p_{\mu}\right) \geq \min _{\tilde{\mu}} D\left(q_{\nu} \| p_{\tilde{\mu}}\right)$ we deduce

$$
F(\boldsymbol{\alpha}) \geq \sum_{\nu=1}^{L} \gamma(\nu) \min _{\tilde{\mu}} D\left(q_{\nu} \| p_{\tilde{\mu}}\right)+\sum_{\nu=1}^{L} \gamma(\nu) H\left(q_{\nu}\right) .
$$

This lower bound is saturated when $\alpha(\nu, \mu)=\gamma(\nu) \mathbb{1}\left[\mu=\operatorname{argmin}_{\tilde{\mu}} D\left(q_{\nu} \| p_{\tilde{\mu}}\right)\right]$ for all $(\mu, \nu)$. For $K \leq L$, this state can be seen as the result of the following 'macroscopic' clustering protocol: for all $\nu \in\{1, \ldots, L\}$, find the distribution $p\left(\mathbf{x} \mid \boldsymbol{\theta}_{\mu}\right)$ with the smallest distance $D\left(q_{\nu} \| p_{\mu}\right)$ to $q_{\nu}(\mathbf{x})$, and assign all members of $\nu$ to cluster $\mu$. If $K<L$ this recipe will occasionally result in the data from more than one distribution being assigned to the same clusters, see Figure 1 (a), but for $K=L$, each cluster would hold only one distribution. Hence, the protocol is able to recover the true partitioning even when the distributions $q_{\nu}(\mathbf{x})$ and $p\left(\mathbf{x} \mid \boldsymbol{\theta}_{\mu}\right)$ are non-identical.

The inequality $D\left(q_{\nu} \| p_{\mu}\right) \geq \min _{\tilde{\nu}} D\left(q_{\tilde{\nu}} \| p_{\mu}\right)$ gives the lower bound

$$
F(\boldsymbol{\alpha}) \geq \sum_{\mu=1}^{K} \alpha(\mu) \min _{\tilde{\nu}} D\left(q_{\tilde{\nu}} \| p_{\mu}\right)+\sum_{\nu=1}^{L} \gamma(\nu) H\left(q_{\nu}\right),
$$

which is saturated when $\alpha(\nu, \mu)=\alpha(\mu) \mathbb{1}\left[\nu=\operatorname{argmin}_{\tilde{\nu}} D\left(q_{\tilde{\nu}} \| p_{\mu}\right)\right]$ for all $(\mu, \nu)$. This state would result from to the following protocol: for all $\nu \in\{1, \ldots, L\}$, find the distribution $p\left(\mathbf{x} \mid \boldsymbol{\theta}_{\mu}\right)$ with the smallest distance $D\left(q_{\nu}|| p_{\mu}\right)$ to $q_{\nu}(\mathbf{x})$, and assign all members of $\mu$ to cluster $\nu$. For $K>L$, this algorithm could allocate more than one distribution to the same 

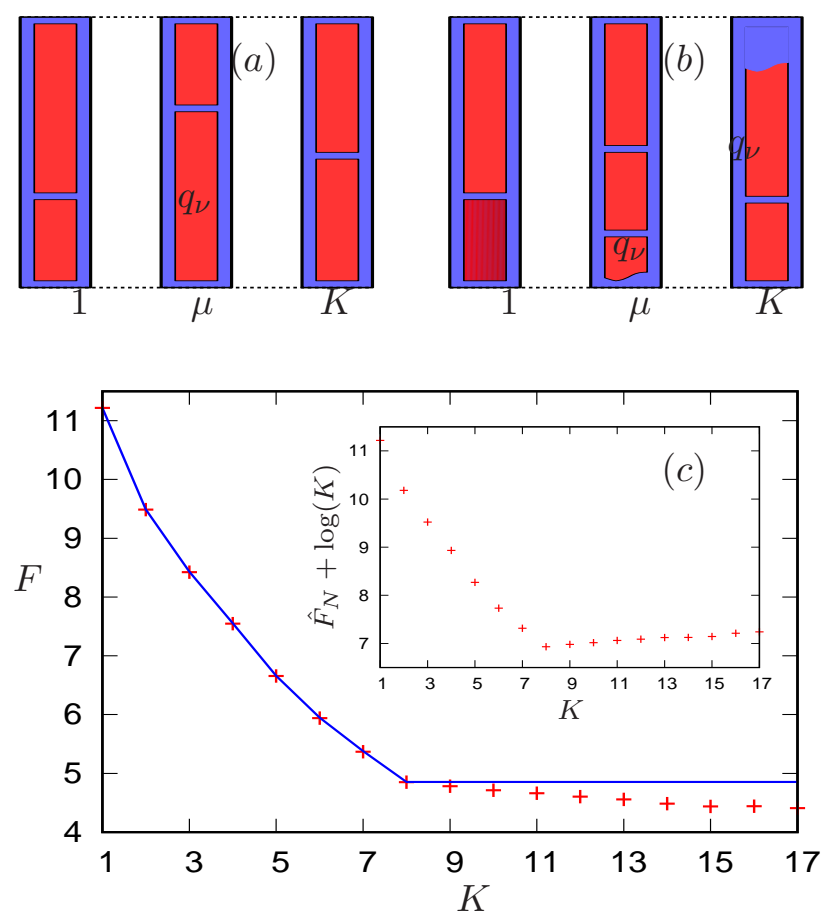

FIG. 1. (Color online) Bayesian clustering: data (red rectangles) from $L$ different distributions $q_{\nu}(\mathbf{x})$ are allocated to $K$ clusters (blue rectangles). a) For $K \leq L$, data from $q_{\nu}(\mathbf{x})$ occupy at most one cluster $\mu$. b) For $K>L$, data from $q_{\nu}(\mathbf{x})$ occupy at least one cluster. c) Minimum $F \equiv \min _{\boldsymbol{\alpha}} F(\boldsymbol{\alpha})$ of the mean-field entropy (blue line), shown as a function of $K$ and compared with the ground state entropy $\hat{F}_{N} \equiv \min _{\mathbf{c}} \hat{F}_{N}(\mathbf{c}, \mathbf{X}$ ) (red crosses), computed for the data of Figure 2 , The horizontal line corresponds to the lower bound $\sum_{\nu \leq L} \gamma(\nu) H\left(q_{\nu}\right)=4.853905$. Inset: the sum of $\hat{F}_{N}$ and $\log (K)$ shown as a function of $K$. The minimum of this sum is obtained when $K=L$. cluster, see Figure 1(b). Furthermore, since $\sum_{\nu=1}^{L} \alpha(\mu) \mathbb{1}\left[\nu=\operatorname{argmin}_{\tilde{\nu}} D\left(q_{\tilde{\nu}} \| p_{\mu}\right)\right]=\alpha(\mu)$, the properties of $\alpha(\nu, \mu)$ imply validity of the set of $L$ linear equations $\sum_{\mu=1}^{K} \alpha(\mu) \mathbb{1}[\nu=$ $\left.\operatorname{argmin}_{\tilde{\nu}} D\left(q_{\tilde{\nu}} \| p_{\mu}\right)\right]=\gamma(\nu)$, which is underdetermined and hence has either infinitely many solutions, or no solutions at all.

We now consider the case where the cluster parameters are unknown, and $p\left(\boldsymbol{\theta}_{\mu}\right)>0$ for all $\left\{\boldsymbol{\theta}_{\mu}\right\}$. For $N \rightarrow \infty$, the entropy (3) is now strictly dominated via steepest descent by the following set of saddle point equations (see Appendix B for details):

$$
\frac{\partial}{\partial \theta_{\mu}(\ell)} \frac{1}{N} \sum_{i=1}^{N} c_{i \mu} \log p\left(\mathbf{x}_{i} \mid \boldsymbol{\theta}_{\mu}\right)=0 .
$$

Solving (12) for Gaussian distributions $p\left(\mathbf{x}_{i} \mid \boldsymbol{\theta}_{\mu}\right) \equiv \mathcal{N}\left(\mathbf{x} \mid \mathbf{m}_{\mu}, \boldsymbol{\Lambda}_{\mu}^{-1}\right)$, with mean $\mathbf{m}_{\mu}$ and inverse 
covariance matrix $\boldsymbol{\Lambda}_{\mu}$, gives us (see Appendix $\mathrm{B}$ ) :

$$
\hat{F}_{N}(\mathbf{c}, \mathbf{X})=\sum_{\mu=1}^{K} \frac{M_{\mu}(\mathbf{c})}{2 N} \log \left((2 \pi \mathrm{e})^{d}\left|\boldsymbol{\Lambda}_{\mu}^{-1}(\mathbf{c}, \mathbf{X})\right|\right),
$$

where $\boldsymbol{\Lambda}_{\mu}^{-1}(\mathbf{c}, \mathbf{X})$ is the empirical covariance matrix of the data in cluster $\mu$.

Since $\frac{1}{2} \log \left((2 \pi \mathrm{e})^{d}\left|\boldsymbol{\Lambda}_{\mu}^{-1}(\mathbf{c}, \mathbf{X})\right|\right)$ is the differential entropy [15] of a Gaussian distribution with covariance matrix $\boldsymbol{\Lambda}_{\mu}^{-1}(\mathbf{c}, \mathbf{X})$, (13) represents an average of $K$ entropies of Gaussian distributions, which for $N \rightarrow \infty$ will converge to the following mean-field entropy (see Appendix C):

$$
F(\boldsymbol{\alpha})=\sum_{\mu=1}^{K} \alpha(\mu) \frac{1}{2} \log \left((2 \pi \mathrm{e})^{d}\left|\boldsymbol{\Lambda}_{\mu}^{-1}(\boldsymbol{\alpha})\right|\right),
$$

in which $\boldsymbol{\Lambda}_{\mu}^{-1}(\boldsymbol{\alpha})$ denotes the covariance matrix

$$
\boldsymbol{\Lambda}_{\mu}^{-1}(\boldsymbol{\alpha})=\sum_{\nu=1}^{L} \alpha(\nu \mid \mu)\left\langle\left(\mathbf{x}-\mathbf{m}_{\mu}(\boldsymbol{\alpha})\right)\left(\mathbf{x}-\mathbf{m}_{\mu}(\boldsymbol{\alpha})\right)^{T}\right\rangle_{\nu}
$$

with $\mathbf{m}_{\mu}(\boldsymbol{\alpha})=\sum_{\nu=1}^{L} \alpha(\nu \mid \mu)\langle\mathbf{x}\rangle_{\nu}$, and the short-hand $\langle\{\cdots\}\rangle_{\nu}=\int \mathrm{d} \mathbf{x} q_{\nu}(\mathbf{x})\{\cdots\}$. Note that (14) also equals

$$
F(\boldsymbol{\alpha})=\sum_{\mu, \nu} \alpha(\nu, \mu) D\left(q_{\nu}|| \mathcal{N}_{\mu}(\boldsymbol{\alpha})\right)+\sum_{\nu=1}^{L} \gamma(\nu) H\left(q_{\nu}\right),
$$

where $\mathcal{N}_{\mu}(\boldsymbol{\alpha}) \equiv \mathcal{N}\left(\mathbf{x} \mid \mathbf{m}_{\mu}(\boldsymbol{\alpha}), \boldsymbol{\Lambda}_{\mu}^{-1}(\boldsymbol{\alpha})\right)$. Moreover, as shown in Appendix D,

$$
F(\boldsymbol{\alpha}) \geq \sum_{\mu=1}^{K} \alpha(\mu) H\left(Q_{\mu}\right) \geq \sum_{\nu=1}^{L} \gamma(\nu) H\left(q_{\nu}\right)
$$

The second inequality in (17) has two consequences. First, if $K \leq L$ then for any state $\boldsymbol{\alpha}$ that corresponds to either of the scenarios depicted in Figures 1 (a,b), we will have $F(\boldsymbol{\alpha}) \geq \min _{K} \min _{\tilde{\boldsymbol{\alpha}}} F(\tilde{\boldsymbol{\alpha}})=\sum_{\nu \leq L} \gamma(\nu) H\left(q_{\nu}\right)$. The lower bound is satisfied when $L=K$ and $q_{\nu}(\mathbf{x})$ is Gaussian. The 'true' parameters $\boldsymbol{\alpha}$ thus represent a locally stable state. Second, when $K>L$, the entropy $F(\boldsymbol{\alpha})$ can only increase with $L$. This follows from (16) and $D\left(q_{\nu} \| \mathcal{N}_{\mu}(\boldsymbol{\alpha})\right) \geq 0$. If $q_{\nu}(\mathbf{x})$ is not Gaussian, then $F(\boldsymbol{\alpha}) \geq \sum_{\nu=1}^{L} \gamma(\nu) \frac{1}{2} \log \left((2 \pi \mathrm{e})^{d}\left|\mathbf{C}_{\nu}\right|\right)$, where $\mathbf{C}_{\nu}$ is the covariance matrix of $q_{\nu}(\mathbf{x})$ (see Appendix D). Equality corresponds to the state shown in the Figure1(a) with $L=K$, i.e. here the 'true' data partitioning is recovered.

The first inequality in (17) has an appealing geometric interpretation. The entropy $H\left(Q_{\mu}\right)$ of each cluster $\mu$ can for large $N$ be estimated by $\left(d / M_{\mu}(\mathbf{c})\right) \sum_{i=1}^{N} c_{i \mu} \log \rho_{i \mu}(\mathbf{c})+$ 
$\log \left(M_{\mu}(\mathbf{c})-1\right)+$ const., where $\rho_{i \mu}(\mathbf{c})=\min _{i \in S_{\mu}(\mathbf{c}) \backslash i}\left\|\mathbf{x}_{i}-\mathbf{x}_{j}\right\|$ (i.e. the Euclidean distance between particle $i$ and its nearest neighbour) [16]. The average entropy $\sum_{\mu=1}^{K} \alpha(\mu) H\left(Q_{\mu}\right)$ is hence estimated by $(d / N) \sum_{\mu=1}^{K} \sum_{i=1}^{N} c_{i \mu} \log \rho_{i \mu}(\mathbf{c})+\sum_{\mu=1}^{K}\left(M_{\mu}(\mathbf{c}) / N\right) \log \left(M_{\mu}(\mathbf{c}) / N\right)+$ const. This is minimized by any state $\mathbf{c}$ which simultaneously maximises the entropy $-\sum_{\mu=1}^{K}\left(M_{\mu}(\mathbf{c}) / N\right) \log \left(M_{\mu}(\mathbf{c}) / N\right)$, i.e. 'disperses' particles maximally over clusters, and minimizes the nearest neighbour distances $\left\{\rho_{i \mu}(\mathbf{c})\right\}$, i.e. favours high particle 'densities' in each cluster.

The lower bound $\sum_{\nu=1}^{L} \gamma(\nu) H\left(q_{\nu}\right)$ in (17) is saturated upon choosing any bijective map $\alpha: \nu \rightarrow \mu$, since this immediately gives us $F(\boldsymbol{\alpha})=\sum_{\nu=1}^{L} \gamma(\nu) H\left(q_{\nu}\right)$. Such maps are special instances of the more general family

$$
\alpha(\nu \mid \mu)=\frac{\mathbb{1}\left[\nu \in S_{\mu}\right] \gamma(\nu)}{\sum_{\tilde{\nu} \in S_{\mu}} \gamma(\tilde{\nu})},
$$

where $\Pi=\left\{S_{1}, \ldots, S_{K}\right\}$ is any partitioning of $[L]$ into $K$ subsets. Finding $\min _{\boldsymbol{\alpha}} F(\boldsymbol{\alpha})$ over all possible matrices of the form (18) by enumeration of all partitions of $[L]$ into $K$ subsets is feasible only for small $L$, since the number of such partitions is given by the Stirling number of the second kind $\mathcal{S}(L, K)$ which grows as $K^{L}$ for large $L[17]$.

One can also compute $\min _{\boldsymbol{\alpha}} F(\boldsymbol{\alpha})$ via the following 'greedy' algorithm. Start with any partition $\Pi$ and compute $F(\boldsymbol{\alpha})$. For all $x \in[L]$ : consider all possible moves which do not create empty clusters, and execute the one which gives the largest decrease in $F(\boldsymbol{\alpha})$, then update $\Pi$. Continue the last two steps until convergence of $F(\boldsymbol{\alpha})$ is observed. This macroscopic algorithm can also be implemented 'microscopically'. At each step: for all $i \in[N]$, consider all possible moves of the particle $i$ from its current cluster $S_{\mu}(\mathbf{c})$ to a new cluster $S_{\nu}(\mathbf{c})$ and select the one which reduces $\hat{F}_{N}(\mathbf{c}, \mathbf{X})$ most. To evolve from a non-ordered state as in Figure 1(b) to an 'ordered' state as in Figure 1(a), this microscopic algorithm has to move on average at least $N(K-1) / K$ particles (see Appendix E). Each move was selected from among $N(K-1)$ possible moves, so the numerical complexity is at least of order $N^{2}(K-1)^{2} / K$.

\section{RESULTS OF NUMERICAL EXPERIMENTS}

Our mean-field theory for was derived under the assumption that $\hat{F}_{N}(\mathbf{c}, \mathbf{X})$ is selfaveraging for $N \rightarrow \infty$. To investigate the correctness of its predictions for finite sample 


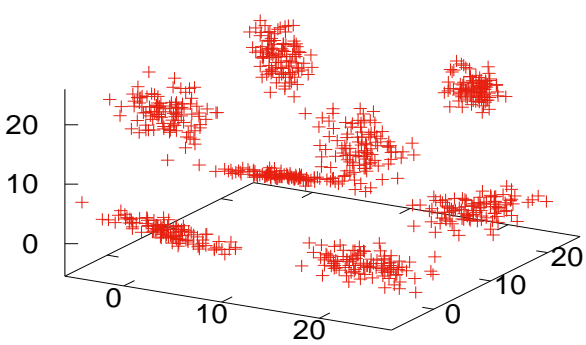

FIG. 2. (Color online) Data used in our numerical experiments. We generated $L=8$ clusters with 1000 data-points each, of which 100 are shown here. The data in each cluster $(i, j, k)$ are generated from a distinct Gaussian distribution, with mean $(\Delta i, \Delta j, \Delta k)$, where $i, j, k \in\{0,1\}(\Delta=20)$, and with covariance matrix sampled from the Wishart distribution with 4 degrees of freedom and precision matrix $\mathbf{1}$.

sizes $N$, we studied low entropy states of (13) as obtained by the gradient descent algorithm on the data of the Figure 2, For each $K \in[17]$ we ran the algorithm from 100 different random initial states $\mathbf{c}(0)$, and computed $\hat{F}_{N}(\mathbf{c}(\infty), \mathbf{X})$ and the mean field entropy $F(\boldsymbol{\alpha})$ (14) for each.

For $K \leq L$, most final states $\mathbf{c}(\infty)$ allocate data from the same distribution correctly to the same cluster, see Figure 1 (a). The values of $\hat{F}_{N}(\mathbf{c}(\infty), \mathbf{X})$ are those predicted by $F(\boldsymbol{\alpha})$, and indeed correspond to local minima and saddle points of $F(\boldsymbol{\alpha})$ (see Appendix F). Also, according to Figure 1 (c), the value $\hat{F}_{N}=\min _{\mathbf{c}} \hat{F}_{N}(\mathbf{c}, \mathbf{X})$ as estimated from $\mathbf{c}(\infty)$ is predicted accurately by $F=\min _{\boldsymbol{\alpha}} F(\boldsymbol{\alpha})$. Residual differences between $\hat{F}_{N}$ and $F$ reflect finite size effects. These can be computed exactly when $K=L$, and when $\mathbf{c}(\infty)$ represents the true partitioning of the data: the average and variance of $\hat{F}_{N}$ are in that case given by $\sum_{\nu=1}^{L} \gamma(\nu) H\left(q_{\nu}\right)+K d(d+1) / 4 N$ and $d / 2 N$, respectively (see Appendix G). Finally, we note that the number of particles 'moved' by the algorithm in going from $\mathbf{c}(0)$ to $\mathbf{c}(\infty)$ is consistent with the lower bound $N(K-1) / K$, so the algorithmic complexity is quadratic in $N$, see Figure 3 .

If $K>L$, the states $\mathbf{c}(\infty)$ will allocate data from the same distribution to multiple clusters, see Figure 1 (b). Such states are already present for small $K \leq L$, and proliferate as $K$ is increased (see Appendix $\mathrm{F}$ ). The lower bound $\sum_{\nu=1}^{L} \gamma(\nu) H\left(q_{\nu}\right)$ is now violated, and the gap between this bound and the value of $\hat{F}_{N}$ as obtained by gradient descent increases with $K$, see Figure 1 (c). While some of the $\hat{F}_{N}(\mathbf{c}(\infty), \mathbf{X})$ values are consistent with $F(\boldsymbol{\alpha})$ 


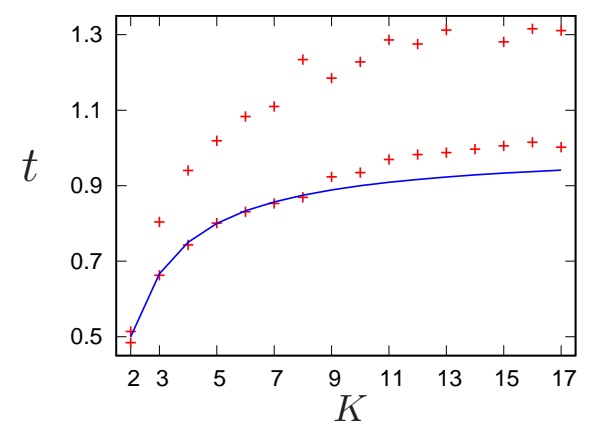

FIG. 3. (Color online) Total (normalised) number of 'moves' $t$ used by the gradient descent algorithm to travel from a random unbiased partition to a final partition, i.e. the effective algorithmic runtime, shown as a function of the assumed number of clusters $K$. The minimum and maximum time (red crosses) obtained in 100 runs on the data of Figure 2 are compared with the average lower bound $(K-1) / K$ (blue line).

(see Appendix $(\mathrm{F})$, the mean-field theory fails to predict $\min _{\mathbf{c}} \hat{F}_{N}(\mathbf{c}, \mathbf{X})$ in this regime, due to the non-commutation of the $N \rightarrow \infty$ limit and the min operator.

Our estimate of $\hat{F}_{N}=\min _{\mathbf{c}} \hat{F}_{N}(\mathbf{c}, \mathbf{X})$ can also be used to infer the true number of clusters $L$. Assuming uniform prior distributions of partitions $p(\mathbf{c} \mid K)=(K ! \mathcal{S}(L, K))^{-1}$ and cluster

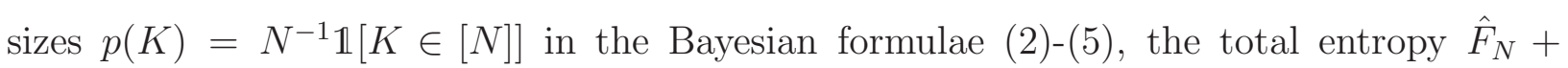
$\frac{1}{N} \log (K ! \mathcal{S}(L, K)) \approx \hat{F}_{N}+\log (K)$ has its minimum at the correct value $K=L$, see inset in Figure 1 (c).

An interesting and important question, from a practical and a theoretical point view, is how Bayesian clustering is affected by the 'separation' between different clusters. The simplest non-trivial case is to consider the clustering of $d$-dimensional data sampled from two isotropic Gaussian distributions $\mathcal{N}\left(\mathbf{m}_{1}, \mathbf{1}\right)$ and $\mathcal{N}\left(\mathbf{m}_{2}, \mathbf{1}\right)$. Here one can use the Euclidean distance $\left\|\mathbf{m}_{1}-\mathbf{m}_{2}\right\|=\Delta$, measured relative to the natural scale $\sqrt{d}$, as a measure of the degree of separation [18] between the 'clusters' centred at $\mathbf{m}_{1}$ and $\mathbf{m}_{2}$. For large $d$, most of the vectors $\mathbf{x}$ sampled from $\mathcal{N}(\mathbf{m}, \mathbf{1})$ will be found in the 'sphere' of radius $\sqrt{d}$ centred at $\mathbf{m}$, reflecting 'concentration' phenomena observed for large $d$. In particular if we assume 
that $\mathbf{x}$ is sampled from $\mathcal{N}(\mathbf{m}, \boldsymbol{\Lambda})$, then $\left\langle\|\mathbf{x}-\mathbf{m}\|^{2}\right\rangle=\operatorname{Tr} \boldsymbol{\Lambda}$, and for $\lambda, \epsilon>0$ :

$$
\begin{array}{r}
\operatorname{Prob}\left(\|\mathbf{x}-\mathbf{m}\|^{2} \geq \operatorname{Tr} \boldsymbol{\Lambda}+d \epsilon\right) \\
=\operatorname{Prob}\left(\mathrm{e}^{\frac{\lambda}{2}\|\mathbf{x}-\mathbf{m}\| \|^{2}} \geq \mathrm{e}^{\frac{\lambda}{2}(\operatorname{Tr} \boldsymbol{\Lambda}+d \epsilon)}\right) \\
\leq\left\langle\mathrm{e}^{\frac{\lambda}{2}\|\mathbf{x}-\mathbf{m}\| \|^{2}}\right\rangle \mathrm{e}^{-\frac{\lambda}{2}(\operatorname{Tr} \boldsymbol{\Lambda}+d \epsilon)} \\
=\mathrm{e}^{-\frac{1}{2}(\log |\mathbf{1}-\lambda \boldsymbol{\Lambda}|+\lambda(\operatorname{Tr} \boldsymbol{\Lambda}+d \epsilon))} .
\end{array}
$$

The upper bound in the above expression was obtained using Markov's inequality and properties of Gaussian integrals. For the choice $\boldsymbol{\Lambda}=\mathbf{1}$, the above inequality, after optimising the upper bound with respect to $\lambda$, gives us $\operatorname{Prob}\left(\|\mathbf{x}-\mathbf{m}\|^{2} \geq d(1+\epsilon)\right) \leq \mathrm{e}^{-\frac{d}{2}\left(\log \frac{1}{1+\epsilon}-\epsilon\right)}$.

Let us now consider the MF entropy $\min _{\boldsymbol{\alpha}} F(\boldsymbol{\alpha})$ for the distributions $\mathcal{N}\left(\mathbf{m}_{1}, \mathbf{1}\right)$ and $\mathcal{N}\left(\mathbf{m}_{2}, \mathbf{1}\right)$, with separation $\left\|\mathbf{m}_{1}-\mathbf{m}_{2}\right\|=\Delta$. For the assumed number of clusters $K=1$ this entropy is given by

$$
F_{1}=\frac{d}{2} \log (2 \pi \mathrm{e})+\frac{1}{2} \log \left|\mathbf{1}+\sum_{\nu=1}^{2} \gamma(\nu)\left(\mathbf{m}_{\nu}-\mathbf{m}\right)\left(\mathbf{m}_{\nu}-\mathbf{m}\right)^{T}\right|
$$

where $\mathbf{m}=\sum_{\nu=1}^{2} \gamma(\nu) \mathbf{m}_{\nu}$, and $\gamma(\nu)$ is the fraction of data sampled from $\mathcal{N}\left(\mathbf{m}_{\nu}, \mathbf{1}\right)$. For $K=2$ we obtain

$$
F_{2}=\frac{d}{2} \log (2 \pi \mathrm{e})
$$

which corresponds to the situation where the true clustering of data is recovered. Furthermore, upon choosing $\mathbf{m}_{1}=\mathbf{0}$ and $\gamma(\nu)=\frac{1}{2}$ we obtain $F_{1}=\frac{d}{2} \log (2 \pi \mathrm{e})+\frac{1}{2} \log \left[1+\left(\frac{\Delta}{2}\right)^{2}\right]$. Thus in this case $F_{1} \geq F_{2}$, as required. However, if $\log (2) \geq \frac{1}{2} \log \left[1+\left(\frac{\Delta}{2}\right)^{2}\right]$ then $F_{2}+\log (K) \geq F_{1}$ (note that we minimise $\min _{\boldsymbol{\alpha}} F(\boldsymbol{\alpha})+\log (K)$ to infer true number of clusters), so that here we are unable to recover the correct number $K=2$ of clusters due to the cluster separation $\Delta$ being too small. This happens when $\Delta \leq 2 \sqrt{3} \approx 3.46$. We expect that a similar analysis can be also performed for more general scenarios.

Numerical experiments are in qualitative agreement with the predicted separation boundary $\Delta=2 \sqrt{3}$, as can be seen in Figure 4. In this Figure we also compare the mean-field theory results (20, 21) with the results of numerical simulations. For $K=1$ the discrepancy between theory and simulations is a finite size effect. In contrast, for $K=2$ it is a combination of finite size effects and the inability of the mean-field theory to account for correlations between the data in clusters for small separations $\Delta$. Such correlations are also responsible 

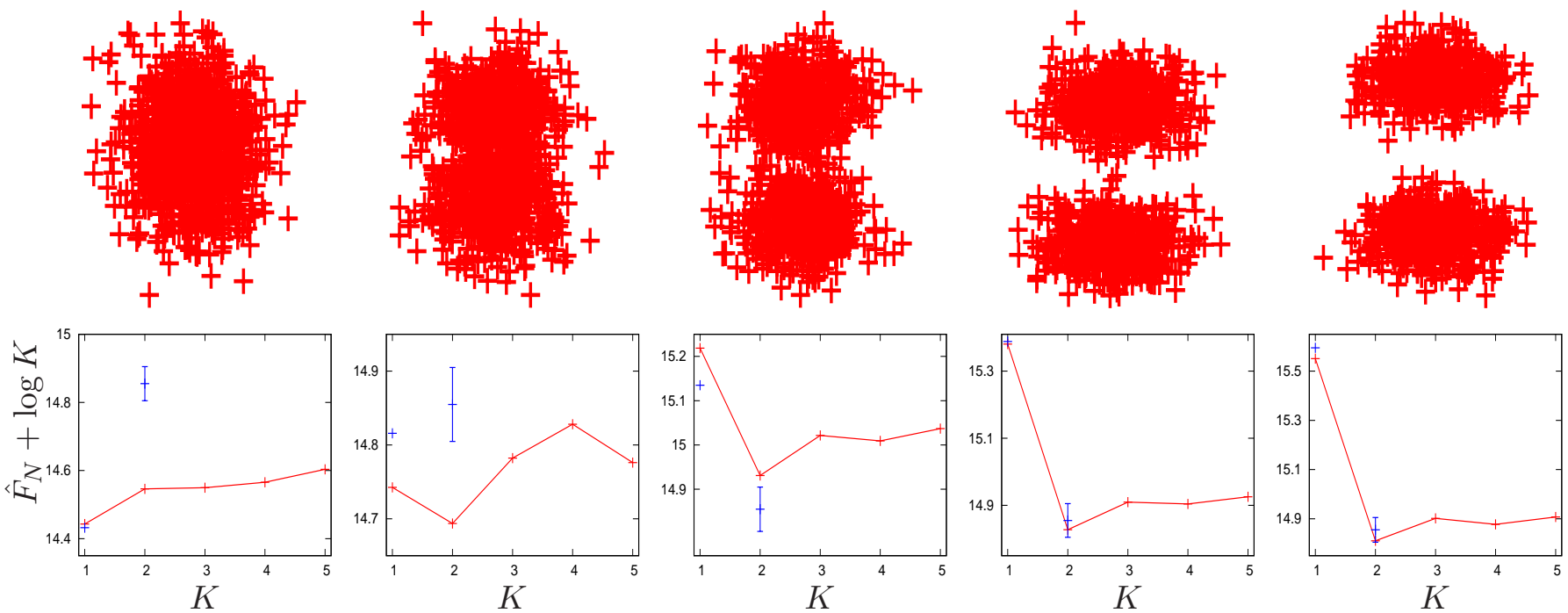

FIG. 4. (Color online) Bayesian clustering of data $\mathbf{x} \in \mathbb{R}^{d}$ generated from the Gaussian distributions $\mathcal{N}\left(\mathbf{m}_{1}, \mathbf{1}\right)$ and $\mathcal{N}\left(\mathbf{m}_{2}, \mathbf{1}\right)$, with separation $\Delta=\left\|\mathbf{m}_{1}-\mathbf{m}_{2}\right\|$. The data sample, split equally between the constituent distributions, is of size $N=2000$ and has $d=10$. The data was generated for cluster separations $\Delta / \sqrt{d} \in\left\{\frac{1}{2}, 1, \frac{3}{2}, 2, \frac{5}{2}\right\}$. Top: Data projected into two dimensions. The separation $\Delta$ of the clusters is increasing from the left to the right. Bottom: the sum $\hat{F}_{N}+\log K$ (red crosses connected by lines), where $\hat{F}_{N} \equiv \min _{\mathbf{c}} \hat{F}_{N}(\mathbf{c}, \mathbf{X})$, shown as a function of the assumed number of clusters $K$, and compared with the mean-field prediction $\min _{\alpha} F(\boldsymbol{\alpha})$ (blue crosses). For $K=2$, the mean-field prediction $\min _{\alpha} F(\boldsymbol{\alpha})=\frac{d}{2} \log (2 \pi \mathrm{e})$ is plotted with the finite size corrections (error bars indicate one standard deviation).

for a breakdown of the mean-field theory when $K>L$, see Figure 1, For larger separations $\Delta$ the theory is in good agreement with the simulations, see Figure 4, and discrepancies again reflect only finite size effects.

The magnitude of the finite size effects can be estimated when $K=L$ for any $d / N<1$, by the following argument. For the empirical covariance matrix $\hat{\Lambda}$ of a sample of $M d$ dimensional data vectors generated from the Gaussian distribution $\mathcal{N}(\mathbf{m}, \boldsymbol{\Lambda})$ the random quantity $\log |\hat{\boldsymbol{\Lambda}}|$ will for large $M$ be described by the distribution

$$
\mathcal{N}\left(\log |\boldsymbol{\Lambda}|+\tau(M, d), \sigma^{2}(M, d)\right)
$$

where $\tau(M, d)=\sum_{\ell=1}^{d} \psi\left(\frac{M-\ell+1}{2}\right)-d \log \left(\frac{M}{2}\right)$ and $\sigma^{2}(M, d)=\sum_{\ell=1}^{d} \frac{2}{M-\ell+1}$ [19]. Assuming that $K=L$ and that the clustering is perfect allows us to compute, by following steps similar to those followed in the Appendix G, the average and variance of the entropy (13). They are 
found to be given by $\min _{\alpha} F(\boldsymbol{\alpha})+\sum_{\nu=1}^{L} \gamma(\nu) \tau(\gamma(\nu) N, d)$ and $\frac{1}{4} \sum_{\nu=1}^{L} \gamma^{2}(\nu) \sigma^{2}(\gamma(\nu) N, d)$, respectively.

When evaluated for real datasets, the entropy function (13) may also have value as an exploratory tool. To show this, we consider the Wisconsin Diagnostic Breast Cancer (WDBC) dataset [20], which describes characteristics of cell nuclei in the images of cells extracted from tumours [21], and contains $N=569$ data-points of dimension $d=30$. This dataset has two (linearly separable) classes, which we assume to be the 'true' clusters, one is 'benign', represented by 357 data-points, and the other is 'malignant', represented by 212 data-points [21]. A first simple unsupervised method which one might apply to this dataset is hierarchical clustering, which uses pairwise distances between the data-points to build a hierarchy of clusters, see e.g. [22]. The agglomerative version of this algorithm, with Euclidean distances, separates this data into clusters of sizes 549 and 20 at the $K=2$ clusters level of hierarchy, into clusters of sizes 549, 19 and 1 at the $K=3$ clusters level of hierarchy, into clusters of sizes 438, 111, 19 and 1 at the $K=4$ clusters level of hierarchy, etc. Hence, upon assuming (correctly) that $K=2$, one cannot recover the true clusters of the WDBC data with this algorithm. Alternatively, the $K$-Means clustering algorithm, see e.g. [5], which minimises the squared Euclidean distance between the points in a cluster, 'finds' in the WDBC dataset (again upon assuming $K=2$ ) clusters of sizes 438 and 131. Upon comparing these with the true clusters, we observe that $K$-Means 'misclassifies' 83 data-points in total. It is interesting that the clusters found by $K$-Means were also present in the four clusters generated via hierarchical clustering.

Using instead the gradient descent minimisation of (13) as a clustering protocol suggests that there are more than $K=4$ clusters 1 in the WDBC dataset (see Figure 5 ). For $K=2$ the algorithm outputs clusters of sizes 328 and 241, which is, compared with the hierarchical and $K$-Means results, much closer to the true sizes 357 and 212 of the WDBC dataset. Now 57 data-points were misclassified, which can be explained by the non-sphericity of clusters in this dataset. In particular, for any data covariance matrix $\hat{\Sigma}$ the ratio $\mathcal{S}(\hat{\Sigma})=\operatorname{Tr}^{2}(\hat{\Sigma}) / d \operatorname{Tr}\left(\hat{\Sigma}^{2}\right)$ can be used as a measure of 'sphericity' of data, see e.g. [23]. We note that $1 / d \leq \mathcal{S}(\hat{\Sigma}) \leq 1$, and that the lower bound $1 / d$ is saturated only when a few eigenvalues dominate all others for large $d$, i.e. when only a few 'directions' in $\mathbb{R}^{d}$ contribute to the variability in the data. The

$\overline{{ }^{1} \text { For } K}>4$, this approach favours small clusters, i.e. we are in non-asymptotic regime, which suggests that a full Bayesian framework is more appropriate for this data. 


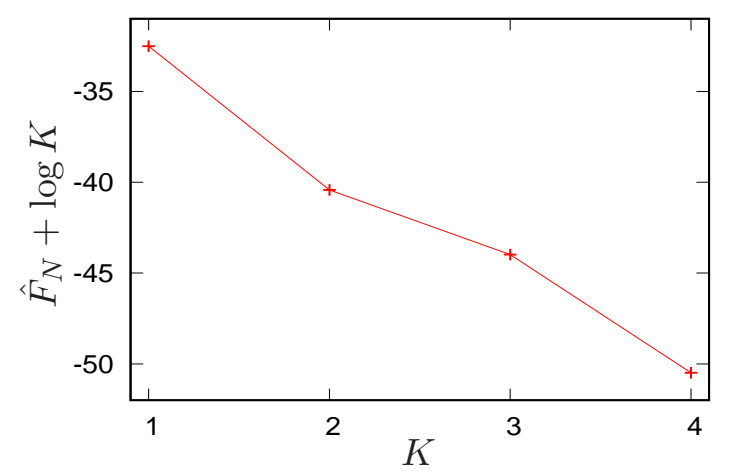

FIG. 5. (Color online) The sum $\hat{F}_{N}+\log K$, where $\hat{F}_{N} \equiv \min _{\mathbf{c}} \hat{F}_{N}(\mathbf{c}, \mathbf{X})$, as computed for the Wisconsin Diagnostic Breast Cancer data 20] (red crosses connected by lines), shown as a function of the assumed number of clusters $K$. These results suggest that the true number of clusters in this dataset is at least $K=4$.

upper bound is saturated when all eigenvalues are equal, i.e. all directions in $\mathbb{R}^{d}$ contribute equally to the variability. The sphericity values of the 'benign' and 'malignant' clusters in the WDBC dataset are given by 0.034 and 0.036 , respectively, so the data in these clusters is highly non-spherical. This indeed suggests that the entropy function (13), derived upon assuming arbitrary multivariate Gaussian distributions of a data in the clusters, is better equipped to deal with this scenario than hierarchical or $K$-Means clustering.

\section{SUMMARY}

In conclusion, in this paper we have demonstrated that mapping Bayesian clustering of data to a statistical mechanical problem is not only possible, but in fact also quite intuitive and fruitful. It enables us to identify objectively the most plausible number of clusters in a dataset, and to obtain transparent interpretations and explanations of why and how conventional clustering methods (which are quite often based on ad-hoc definitions) may or may not fail to detect clusters correctly, dependent on the quantitative features of the data.

One possible extension of this work, currently in progress, is a more general analytical treatment of this Bayesian clustering problem, in which the distribution $P(F)=$ $\int \mathrm{d} \boldsymbol{\alpha} P(\boldsymbol{\alpha}) \delta(F-F(\boldsymbol{\alpha}))$ is no longer assumed to converge to a delta distribution for large $N$. This will allow us allow us to tackle also the nontrivial regime where $N, d \rightarrow \infty$ with $N / d$ finite, and to correct the present mean-field theory in the $K>L$ regime. 


\section{ACKNOWLEDGMENTS}

This work was supported by the Medical Research Council of the United Kingdom (grant MR/L01257X/1).

\section{Appendix A: Model of data and Bayesian clustering}

Let us assume that we observe the sample $\mathbf{X}=\left\{\mathbf{x}_{1}, \ldots, \mathbf{x}_{N}\right\}$, where $\mathbf{x}_{i} \in \mathbb{R}^{d}$ for all $i$, drawn from the distribution

$$
p(\mathbf{X} \mid \boldsymbol{\Theta}, \Pi)=\prod_{\mu=1}^{|\Pi|} \prod_{i_{\mu} \in S_{\mu}} p\left(\mathbf{x}_{i_{\mu}} \mid \boldsymbol{\theta}_{\mu}\right),
$$

generated by the partition $\Pi=\left\{S_{1}, S_{2}, \ldots, S_{|\Pi|}\right\}$, where the index sets $S_{\mu} \neq \emptyset$ obey $S_{\mu} \cap S_{\nu}=$ $\emptyset$ for $\mu \neq \nu$, and $\cup_{\mu=1}^{|\Pi|} S_{\mu}=[N]$, with the short-hand $[N]=\{1, \ldots, N\}$. Furthermore, we assume that each parameter $\boldsymbol{\theta}_{\mu}$ is sampled randomly and independently from the distribution $p\left(\boldsymbol{\theta}_{\mu}\right)$, and that we are also given the prior distribution of $\Pi, P(\Pi)$. This allows us to write down the joint distribution

$$
p(\mathbf{X}, \boldsymbol{\Theta}, \Pi)=p(\mathbf{X} \mid \boldsymbol{\Theta}, \Pi) p(\Pi) \prod_{\mu=1}^{|\Pi|} p\left(\boldsymbol{\theta}_{\mu}\right),
$$

where $\boldsymbol{\Theta}=\left\{\boldsymbol{\theta}_{1}, \ldots, \boldsymbol{\theta}_{|\Pi|}\right\}$. Upon integrating out the parameters $\boldsymbol{\theta}_{\mu}$ in the above we obtain the distribution

$$
p(\mathbf{X}, \Pi)=\langle p(\mathbf{X} \mid \mathbf{\Theta}, \Pi)\rangle_{\Theta \mid \Pi} p(\Pi),
$$

where $\langle f(\boldsymbol{\Theta})\rangle_{\boldsymbol{\Theta} \mid \Pi}=\int f(\boldsymbol{\Theta})\left\{\prod_{\mu=1}^{|\Pi|} p\left(\boldsymbol{\theta}_{\mu}\right) \mathrm{d} \boldsymbol{\theta}_{\mu}\right\}$. From this follows the conditional distribution

$$
p(\Pi \mid \mathbf{X})=\frac{p(\mathbf{X} \mid \Pi) p(\Pi)}{\sum_{\tilde{\Pi}} p(\mathbf{X} \mid \tilde{\Pi}) p(\tilde{\Pi})}
$$

with

$$
p(\mathbf{X} \mid \Pi)=\langle p(\mathbf{X} \mid \boldsymbol{\Theta}, \Pi)\rangle_{\boldsymbol{\Theta} \mid \Pi} .
$$


Let us next consider the 'partition function'

$$
\begin{aligned}
\sum_{\Pi} p & (\mathbf{X} \mid \Pi) p(\Pi) \\
= & \sum_{K=1}^{N} \sum_{\Pi} p(\mathbf{X} \mid \Pi) p(\Pi) \mathbb{1}[|\Pi|=K] \\
= & \sum_{K=1}^{N} \sum_{\Pi} p(\mathbf{X} \mid \Pi) p(\Pi \mid K) p(K)
\end{aligned}
$$

where we have defined the two distributions

$$
\begin{aligned}
p(\Pi \mid K) & =\frac{p(\Pi) \mathbb{1}[|\Pi|=K]}{\sum_{\tilde{\Pi}} p(\tilde{\Pi}) \mathbb{1}[|\tilde{\Pi}|=K]} \\
p(K) & =\sum_{\Pi} p(\Pi) \mathbb{1}[|\Pi|=K] .
\end{aligned}
$$

Furthermore, if we define $\Pi_{K}$ to be a partition $\Pi$ with $|\Pi|=K$, i.e. $\Pi_{K}=\left\{S_{1}, \ldots, S_{K}\right\}$, then

$$
\begin{aligned}
\sum_{\Pi} p & (\mathbf{X} \mid \Pi) p(\Pi) \\
= & \sum_{K=1}^{N} p(K) \sum_{\Pi_{K}} p\left(\mathbf{X} \mid \Pi_{K}\right) p\left(\Pi_{K} \mid K\right)
\end{aligned}
$$

and the distribution of $\Pi_{K}$ is given by

$$
p\left(\Pi_{K} \mid(\mathbf{X})=\frac{p\left(\mathbf{X} \mid \Pi_{K}\right) p\left(\Pi_{K} \mid K\right) p(K)}{\sum_{\tilde{K}=1}^{N} p(\tilde{K}) \sum_{\tilde{\Pi}_{\tilde{K}}} p\left(\mathbf{X} \mid \tilde{\Pi}_{\tilde{K}}\right) p\left(\tilde{\Pi}_{\tilde{K}} \mid \tilde{K}\right)} .\right.
$$

The mode of this distribution is located at

$$
\hat{\Pi}_{K}=\operatorname{argmax}_{\Pi_{K}}\left\{p\left(\mathbf{X} \mid \Pi_{K}\right) p\left(\Pi_{K} \mid K\right)\right\} .
$$

from which, in turn, it follows that the mode of the distribution (A4) is located at

$$
\hat{\Pi}=\operatorname{argmax}_{\hat{\Pi}_{K}}\left\{p\left(\mathbf{X} \mid \hat{\Pi}_{K}\right) p\left(\hat{\Pi}_{K} \mid K\right) p(K)\right\} .
$$

To see this one considers

$$
\begin{gathered}
\hat{\Pi}=\operatorname{argmax}_{\Pi}\{p(\mathbf{X} \mid \Pi) p(\Pi)\} \\
=\operatorname{argmax}_{\Pi}\left\{\left\{p\left(\mathbf{X} \mid \Pi_{1}\right) p\left(\Pi_{1}\right)\right\}, \ldots\right. \\
\ldots,\left\{p\left(\mathbf{X} \mid \Pi_{K}\right) p\left(\Pi_{K}\right)\right\}, \ldots \\
\left.\ldots,\left\{p\left(\mathbf{X} \mid \Pi_{N}\right) p\left(\Pi_{N}\right)\right\}\right\},
\end{gathered}
$$


where $\left\{p\left(\mathbf{X} \mid \Pi_{K}\right) p\left(\Pi_{K}\right)\right\}$ is a set generated by $\left\{\Pi_{K}\right\}$. Clearly, $\max _{\Pi_{K}}\left\{p\left(\mathbf{X} \mid \Pi_{K}\right) p\left(\Pi_{K}\right)\right\}=$ $p\left(\mathbf{X} \mid \hat{\Pi}_{K}\right) p\left(\hat{\Pi}_{K}\right)$, in which $\hat{\Pi}_{K}=\operatorname{argmax}_{\Pi_{K}}\left\{p\left(\mathbf{X} \mid \Pi_{K}\right) p\left(\Pi_{K}\right)\right\}$, from which follows that

$$
\begin{aligned}
\hat{\Pi} & =\operatorname{argmax}_{\hat{\Pi}_{K}}\left\{p\left(\mathbf{X} \mid \hat{\Pi}_{K}\right) p\left(\hat{\Pi}_{K}\right)\right\} \\
& =\operatorname{argmax}_{\hat{\Pi}_{K}}\left\{p\left(\mathbf{X} \mid \hat{\Pi}_{K}\right) p\left(\hat{\Pi}_{K} \mid K\right) p(K)\right\} .
\end{aligned}
$$

Any partition $\Pi_{K}$ of the data into $K$ clusters can be specified by the binary 'allocation' variables $c_{i \mu}=\mathbb{1}\left[i \in S_{\mu}\right]$, where $i \in[N]$ and $\mu \in[K]$, forming the matrix $\mathbf{c}$ with $[\mathbf{c}]_{i \mu}=c_{i \mu}$. Hence $\Pi_{K} \equiv \Pi_{K}(\mathbf{c})=\left\{S_{1}(\mathbf{c}), \ldots, S_{K}(\mathbf{c})\right\}$. Conversely, an $N \times K$ matrix c with binary entries is a partition only if it satisfies the constraints $\sum_{\mu=1}^{K} c_{i \mu}=1$ for all $i \in[N]$ and $\sum_{i=1}^{N} c_{i \mu} \geq 1$ for all $\mu \in[K]$. The simplest distribution implementing these constraints is the uniform distribution

$$
p(\mathbf{c} \mid K)=\frac{\left\{\prod_{i=1}^{N} \mathbb{1}\left[\sum_{\nu=1}^{K} c_{i \nu}=1\right]\right\}\left\{\prod_{\mu=1}^{K} \mathbb{1}\left[\sum_{j=1}^{N} c_{j \mu} \geq 1\right]\right\}}{\sum_{\tilde{\mathbf{c}}}\left\{\prod_{i=1}^{N} \mathbb{1}\left[\sum_{\nu=1}^{K} \tilde{c}_{i \nu}=1\right]\right\}\left\{\prod_{\mu=1}^{K} \mathbb{1}\left[\sum_{j=1}^{N} \tilde{c}_{j \mu} \geq 1\right]\right\}} .
$$

The denominator in this expression gives the total number of partitions of the set $[N]$ into $K$ subsets $\mathcal{S}(N, K)$, i.e. it equals the Stirling number of the second kind times the number $K$ ! of subset permutations. Thus the probability of each individual partition $\mathbf{c}$ is given by $1 / K ! \mathcal{S}(N, K)$. We note that for $N \rightarrow \infty$ and $K \in O\left(N^{0}\right)$ we have $N^{-1} \log (K ! \mathcal{S}(N, K)) \rightarrow$ $\log (K)[17]$.

Using this new notation allows us to write the distribution $p\left(\mathbf{X} \mid \Pi_{K}\right)$ as

$$
\begin{aligned}
p\left(\mathbf{X} \mid \Pi_{K}\right) & \equiv p(\mathbf{X} \mid \mathbf{c}, K) \\
& =\left\langle\mathrm{e}^{\sum_{\mu=1}^{K} \sum_{i=1}^{N} c_{i \mu} \log p\left(\mathbf{x}_{i} \mid \boldsymbol{\theta}_{\mu}\right)}\right\rangle_{\boldsymbol{\Theta}} \\
& =\mathrm{e}^{-N \hat{F}_{N}(\mathbf{c}, \mathbf{X})},
\end{aligned}
$$

where $\langle f(\boldsymbol{\Theta})\rangle_{\boldsymbol{\Theta}}=\int f(\boldsymbol{\Theta})\left\{\prod_{\mu=1}^{K} p\left(\boldsymbol{\theta}_{\mu}\right) \mathrm{d} \boldsymbol{\theta}_{\mu}\right\}$, and we defined the log-likelihood

$$
\hat{F}_{N}(\mathbf{c}, \mathbf{X})=-\frac{1}{N} \log \left\langle\mathrm{e}^{\sum_{\mu=1}^{K} \sum_{i=1}^{N} c_{i \mu} \log p\left(\mathbf{x}_{i} \mid \boldsymbol{\theta}_{\mu}\right)}\right\rangle_{\boldsymbol{\Theta}}
$$

Furthermore, combining $p(\mathbf{c}, K)=p(\mathbf{c} \mid K) p(K)$ with (A14) gives us the joint distribution

$$
p(\mathbf{X}, \mathbf{c}, K)=\mathrm{e}^{-N \hat{F}_{N}(\mathbf{c}, \mathbf{X})} p(\mathbf{c}, K)
$$


from which we can derive the conditional distribution

$$
\begin{aligned}
& p(\mathbf{c}, K \mid \mathbf{X}) \\
& \quad=\frac{\mathrm{e}^{-N \hat{F}_{N}(\mathbf{c}, \mathbf{X})} p(\mathbf{c} \mid K) p(K)}{\sum_{\tilde{K}=1}^{N} p(\tilde{K}) \sum_{\tilde{\mathbf{c}}} \mathrm{e}^{-N \hat{F}_{N}(\tilde{\mathbf{c}}, \mathbf{X})} p(\tilde{\mathbf{c}} \mid \tilde{K})}
\end{aligned}
$$

For $K \in[N]$ the mode of this distribution is located at

$$
\begin{aligned}
\hat{\mathbf{c}} \mid K & =\operatorname{argmax}_{\mathbf{c}} p(\mathbf{c}, K \mid \mathbf{X}) \\
& =\operatorname{argmax}_{\mathbf{c}}\left\{\mathrm{e}^{-N \hat{F}_{N}(\mathbf{c}, \mathbf{X})} p(\mathbf{c} \mid K)\right\}
\end{aligned}
$$

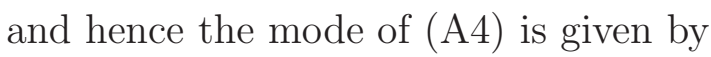

$$
\hat{\Pi}=\operatorname{argmax}_{\hat{\mathbf{c}} \mid K}\left\{\mathrm{e}^{-N \hat{F}_{N}(\mathbf{c}, \mathbf{X})} p(\hat{\mathbf{c}} \mid K) p(K)\right\}
$$

which is our MAP estimator of the partition of data $\Pi$.

\section{Appendix B: Laplace approximation}

Let us consider the log-likelihood density (3). We note that $\hat{F}_{N}(\mathbf{c}, \mathbf{X})=\sum_{\mu=1}^{K} \hat{F}_{\mu}^{N}(\mathbf{c}, \mathbf{X})$, where

$$
\begin{aligned}
\hat{F}_{\mu}^{N}(\mathbf{c}, \mathbf{X}) & =-\frac{1}{N} \log \int \mathrm{e}^{-N \Phi_{\mu}\left(\boldsymbol{\theta}_{\mu} \mid \mathbf{c}, \mathbf{X}\right)} p\left(\boldsymbol{\theta}_{\mu}\right) \mathrm{d} \boldsymbol{\theta}_{\mu} \\
\Phi_{\mu}\left(\boldsymbol{\theta}_{\mu} \mid \mathbf{c}, \mathbf{X}\right) & =-\frac{1}{N} \sum_{i=1}^{N} c_{i \mu} \log p\left(\mathbf{x}_{i} \mid \boldsymbol{\theta}_{\mu}\right)
\end{aligned}
$$

$\hat{F}_{\mu}^{N}(\mathbf{c}, \mathbf{X})$ is a log-likelihood density of cluster $\mu$. For large $N$ it can be evaluated by the Laplace method [24]:

$$
\begin{aligned}
\hat{F}_{\mu}^{N}(\mathbf{c}, \mathbf{X})= & -\frac{1}{N} \log \left(\frac{\int \mathrm{e}^{-N \Phi_{\mu}\left(\boldsymbol{\theta}_{\mu} \mid \mathbf{c}, \mathbf{X}\right)} p\left(\boldsymbol{\theta}_{\mu}\right) \mathrm{d} \boldsymbol{\theta}_{\mu}}{\int \mathrm{e}^{-N \Phi_{\mu}\left(\tilde{\boldsymbol{\theta}}_{\mu} \mid \mathbf{c}, \mathbf{X}\right)} \mathrm{d} \tilde{\boldsymbol{\theta}}_{\mu}} \int \mathrm{e}^{-N \Phi_{\mu}\left(\tilde{\boldsymbol{\theta}}_{\mu} \mid \mathbf{c}, \mathbf{X}\right)} \mathrm{d} \tilde{\boldsymbol{\theta}}_{\mu}\right) \\
& =\Phi_{\mu}\left(\boldsymbol{\theta}_{\mu}^{*} \mid \mathbf{c}\right),
\end{aligned}
$$

where

$$
\boldsymbol{\theta}_{\mu}^{*}=\operatorname{argmin}_{\boldsymbol{\theta}} \Phi_{\mu}(\boldsymbol{\theta} \mid \mathbf{c}, \mathbf{X}) .
$$

The stationarity condition $\frac{\partial}{\partial \theta_{\mu}(\ell)} \Phi_{\mu}(\boldsymbol{\theta} \mid \mathbf{c}, \mathbf{X})=0$ for all $\ell$, from which to solve $\boldsymbol{\theta}_{\mu}^{*}$, gives us the equations

$$
\frac{\partial}{\partial \theta_{\mu}(\ell)} \frac{1}{N} \sum_{i=1}^{N} c_{i \mu} \log p\left(\mathbf{x}_{i} \mid \boldsymbol{\theta}_{\mu}\right)=0 .
$$


Let us now evaluate (B4) for the multivariate Gaussian distributions

$$
\mathcal{N}\left(\mathbf{x} \mid \mathbf{m}_{\mu}, \boldsymbol{\Lambda}_{\mu}^{-1}\right)=\frac{\mathrm{e}^{-\frac{1}{2}\left(\mathbf{x}-\mathbf{m}_{\mu}\right)^{T} \boldsymbol{\Lambda}_{\mu}\left(\mathbf{x}-\mathbf{m}_{\mu}\right)}}{\left|2 \pi \boldsymbol{\Lambda}_{\mu}^{-1}\right|^{\frac{1}{2}}},
$$

with the means $\mathbf{m}_{\mu}$ and the inverse covariance matrices $\boldsymbol{\Lambda}_{\mu}$. Upon assuming that $p\left(\mathbf{x}_{i} \mid \boldsymbol{\theta}_{\mu}\right) \equiv$ $\mathcal{N}\left(\mathbf{x} \mid \mathbf{m}_{\mu}, \boldsymbol{\Lambda}_{\mu}^{-1}\right)$, the desired log-likelihood density becomes

$$
\begin{aligned}
-\frac{1}{N} \sum_{i=1}^{N} c_{i \mu} \log \mathcal{N}\left(\mathbf{x}_{i} \mid \mathbf{m}_{\mu}, \boldsymbol{\Lambda}_{\mu}^{-1}\right) & = \\
& \frac{1}{2 N} \sum_{i=1}^{N} c_{i \mu}\left(\mathbf{x}_{i}-\mathbf{m}_{\mu}\right)^{T} \boldsymbol{\Lambda}_{\mu}\left(\mathbf{x}_{i}-\mathbf{m}_{\mu}\right) \\
& -\frac{M_{\mu}(\mathbf{c})}{2 N} \log \left((2 \pi)^{-d}\left|\boldsymbol{\Lambda}_{\mu}\right|\right)
\end{aligned}
$$

Here $M_{\mu}(\mathbf{c})=\sum_{i=1}^{N} c_{i \mu}=\left|S_{\mu}(\mathbf{c})\right|$ denotes the number of data points in cluster $\mu$. Solving the equations $\frac{\partial}{\partial m_{\mu \ell}} \sum_{i=1}^{N} c_{i \mu} \log \mathcal{N}\left(\mathbf{x}_{i} \mid \mathbf{m}_{\mu}, \boldsymbol{\Lambda}_{\mu}^{-1}\right)=0$ and

$$
\frac{\partial}{\partial\left[\boldsymbol{\Lambda}_{\mu}\right]_{s \ell}} \sum_{i=1}^{N} c_{i \mu} \log \mathcal{N}\left(\mathbf{x}_{i} \mid \mathbf{m}_{\mu}, \boldsymbol{\Lambda}_{\mu}^{-1}\right)=0
$$

gives us

$$
\begin{aligned}
\mathbf{m}_{\mu} & =\frac{1}{M_{\mu}(\mathbf{c})} \sum_{i=1}^{N} c_{i \mu} \mathbf{x}_{i} \\
\boldsymbol{\Lambda}_{\mu}^{-1} & =\frac{1}{M_{\mu}(\mathbf{c})} \sum_{i=1}^{N} c_{i \mu}\left(\mathbf{x}_{i}-\mathbf{m}_{\mu}\right)\left(\mathbf{x}_{i}-\mathbf{m}_{\mu}\right)^{T}
\end{aligned}
$$

i.e. the empirical mean and covariance of the data in cluster $\mu$. Using the above results in equation (B6) we then obtain the log-likelihood density (13).

\section{Appendix C: Distribution of log-likelihood - A 'field theory' approach}

Let us assume that the data $\mathbf{X}=\left\{\mathbf{x}_{1}, \ldots, \mathbf{x}_{N}\right\}$ are sampled from the distribution

$$
p(\mathbf{X} \mid L)=\sum_{\tilde{\mathbf{c}}} q(\tilde{\mathbf{c}} \mid L)\left\{\prod_{\nu=1}^{L} \prod_{i_{\nu} \in S_{\nu}(\tilde{\mathbf{c}})} q_{\nu}\left(\mathbf{x}_{i_{\nu}}\right)\right\},
$$


where $q(\tilde{\mathbf{c}} \mid L)$ is the 'true' distribution of the partitions $\tilde{\mathbf{c}}$ of size $L$. We are interested in computing the distribution of log-likelihoods

$$
\begin{aligned}
P_{N}(F) & =\sum_{\mathbf{c}} p(\mathbf{c} \mid K) \int \mathrm{d} \mathbf{X} p(\mathbf{X} \mid L) \delta\left(F-\hat{F}_{N}(\mathbf{c}, \mathbf{X})\right) \\
\hat{F}_{N}(\mathbf{c}, \mathbf{X}) & =-\frac{1}{N} \sum_{\mu=1}^{K} \sum_{i=1}^{N} c_{i \mu} \log p\left(\mathbf{x}_{i} \mid \boldsymbol{\theta}_{\mu}\right) .
\end{aligned}
$$

Here $p(\mathbf{c} \mid K)$ is our 'assumed' distribution of the partition $\mathbf{c}$ of size $K$. Let us now evaluate $P_{N}(F)$ further:

$$
P_{N}(F)=\sum_{\mathbf{c}, \tilde{\mathbf{c}}} p(\mathbf{c} \mid K) q(\tilde{\mathbf{c}} \mid L) \int\left\{\prod_{\nu=1}^{L} \prod_{i_{\nu} \in S_{\nu}(\tilde{\mathbf{c}})} q_{\nu}\left(\mathbf{x}_{i_{\nu}}\right)\right\} \delta\left(F-\hat{F}_{N}(\mathbf{c}, \mathbf{X})\right) \mathrm{d} \mathbf{X}
$$

We note that the sum over $\tilde{\mathbf{c}}$ inside the function $\hat{F}_{N}(\mathbf{c}, \mathbf{X})$ can be written in the following form

$$
\begin{aligned}
-\hat{F}_{N}(\mathbf{c}, \mathbf{X}) & =\sum_{\mu=1}^{K} \frac{\left|S_{\mu}(\mathbf{c})\right|}{N} \int \frac{1}{\left|S_{\mu}(\mathbf{c})\right|} \sum_{i_{\mu} \in S_{\mu}(\mathbf{c})} \delta\left(\mathbf{x}-\mathbf{x}_{i_{\mu}}\right) \log p\left(\mathbf{x} \mid \boldsymbol{\theta}_{\mu}\right) \mathrm{d} \mathbf{x} \\
& =\sum_{\mu=1}^{K} \frac{\left|S_{\mu}(\mathbf{c})\right|}{N} \int \frac{1}{\left|S_{\mu}(\mathbf{c})\right|} \sum_{\nu=1}^{L} \sum_{i_{\mu \nu} \in S_{\mu}(\mathbf{c}) \cap S_{\nu}(\tilde{\mathbf{c}})} \delta\left(\mathbf{x}-\mathbf{x}_{i_{\mu \nu}}\right) \log p\left(\mathbf{x} \mid \boldsymbol{\theta}_{\mu}\right) \mathrm{d} \mathbf{x} \\
& =\sum_{\mu=1}^{K} \frac{\left|S_{\mu}(\mathbf{c})\right|}{N} \int Q_{\mu}(\mathbf{x} \mid \mathbf{c}, \tilde{\mathbf{c}}, \mathbf{X}) \log p\left(\mathbf{x} \mid \boldsymbol{\theta}_{\mu}\right) \mathrm{d} \mathbf{x},
\end{aligned}
$$

where we have defined the density

$$
Q_{\mu}(\mathbf{x} \mid \mathbf{c}, \tilde{\mathbf{c}}, \mathbf{X})=\frac{1}{\left|S_{\mu}(\mathbf{c})\right|} \sum_{\nu=1}^{L} \sum_{i_{\mu \nu} \in S_{\mu}(\mathbf{c}) \cap S_{\nu}(\tilde{\mathbf{c}})} \delta\left(\mathbf{x}-\mathbf{x}_{i_{\mu \nu}}\right)
$$

Using the above form in (4) we obtain

$$
\begin{aligned}
P_{N}(F)=\sum_{\mathbf{c}, \tilde{\mathbf{c}}} p(\mathbf{c} \mid K) q(\tilde{\mathbf{c}} \mid L) & \int \mathrm{d} \mathbf{X}\left\{\prod_{\nu=1}^{L} \prod_{i_{\nu} \in S_{\nu}(\tilde{\mathbf{c}})} q_{\nu}\left(\mathbf{x}_{i_{\nu}}\right)\right\} \\
\times & \delta\left(F+\sum_{\mu=1}^{K} \frac{\left|S_{\mu}(\mathbf{c})\right|}{N} \int Q_{\mu}(\mathbf{x} \mid \mathbf{c}, \tilde{\mathbf{c}}, \mathbf{X}) \log p\left(\mathbf{x} \mid \boldsymbol{\theta}_{\mu}\right) \mathrm{d} \mathbf{x}\right) \\
=\sum_{\mathbf{c}, \tilde{\mathbf{c}}} p(\mathbf{c} \mid K) q(\tilde{\mathbf{c}} \mid L) & \left\{\prod_{\mu=1}^{K} \prod_{\mathbf{x}} \int \mathrm{d} Q_{\mu}(\mathbf{x})\right\} P_{N}\left[\left\{Q_{\mu}(\mathbf{x})\right\} \mid \mathbf{c}, \tilde{\mathbf{c}}\right] \\
\times & \delta\left(F+\sum_{\mu=1}^{K} \frac{\left|S_{\mu}(\mathbf{c})\right|}{N} \int Q_{\mu}(\mathbf{x}) \log p\left(\mathbf{x} \mid \boldsymbol{\theta}_{\mu}\right) \mathrm{d} \mathbf{x}\right),
\end{aligned}
$$


were we have defined the (functional) distribution

Let us next consider

$$
\begin{aligned}
P_{N}\left[\left\{Q_{\mu}(\mathbf{x})\right\} \mid \mathbf{c}, \tilde{\mathbf{c}}\right]=\int & \left\{\prod_{\nu=1}^{L} \prod_{i_{\nu} \in S_{\nu}(\tilde{\mathbf{c}})} q_{\nu}\left(\mathbf{x}_{i_{\nu}}\right)\right\} \\
& \times\left\{\prod_{\mu=1}^{K} \prod_{\mathbf{x}} \delta\left[Q_{\mu}(\mathbf{x})-Q_{\mu}(\mathbf{x} \mid \mathbf{c}, \tilde{\mathbf{c}}, \mathbf{X})\right]\right\} \mathrm{d} \mathbf{X} .
\end{aligned}
$$

$$
\begin{aligned}
& P_{N}\left[\left\{Q_{\mu}(\mathbf{x})\right\} \mid \mathbf{c}, \tilde{\mathbf{c}}\right] \\
& =\int \mathrm{d} \mathbf{X}\left\{\prod_{\nu=1}^{L} \prod_{i_{\nu} \in S_{\nu}(\tilde{\mathbf{c}})} q_{\nu}\left(\mathbf{x}_{i_{\nu}}\right)\right\}\left\{\prod_{\mu=1}^{K} \prod_{\mathbf{x}} \int \frac{\mathrm{d} \hat{Q}_{\mu}(\mathbf{x})}{2 \pi / N}\right\} \\
& \times \mathrm{e}^{\mathrm{i} N \sum_{\mu=1}^{K} \int \hat{Q}_{\mu}(\mathbf{x})\left[Q_{\mu}(\mathbf{x})-Q_{\mu}(\mathbf{x} \mid \mathbf{c}, \tilde{\mathbf{c}}, \mathbf{X})\right] \mathrm{d} \mathbf{x}} \\
& =\left\{\prod_{\mu=1}^{K} \prod_{\mathbf{x}} \int \frac{\mathrm{d} \hat{Q}_{\mu}(\mathbf{x})}{2 \pi / N}\right\} \mathrm{e}^{\mathrm{i} N \sum_{\mu=1}^{K} \int \hat{Q}_{\mu}(\mathbf{x}) Q_{\mu}(\mathbf{x}) \mathrm{d} \mathbf{x}} \\
& \times \int \mathrm{d} \mathbf{X}\left\{\prod_{\nu=1}^{L} \prod_{i_{\nu} \in S_{\nu}(\tilde{\mathbf{c}})} q_{\nu}\left(\mathbf{x}_{i_{\nu}}\right)\right\} \mathrm{e}^{\sum_{\nu=1}^{L} \sum_{\mu=1}^{K} \frac{N}{\left|S_{\mu}(\mathbf{c})\right|} \sum_{i_{\mu \nu} \in S_{\mu}(\mathbf{c}) \cap S_{\nu}(\tilde{\mathbf{c}})}-\mathrm{i} \hat{Q}_{\mu}\left(\mathbf{x}_{i_{\mu \nu}}\right)} \\
& =\left\{\prod_{\mu=1}^{K} \prod_{\mathbf{x}} \int \frac{\mathrm{d} \hat{Q}_{\mu}(\mathbf{x})}{2 \pi / N}\right\} \mathrm{e}^{\mathrm{i} N \sum_{\mu=1}^{K} \int \hat{Q}_{\mu}(\mathbf{x}) Q_{\mu}(\mathbf{x}) \mathrm{d} \mathbf{x}} \\
& \times \prod_{\nu=1}^{L} \prod_{\mu=1}^{K} \prod_{i_{\mu \nu} \in S_{\mu}(\mathbf{c}) \cap S_{\nu}(\tilde{\mathbf{c}})} \int q_{\nu}\left(\mathbf{x}_{i_{\mu \nu}}\right) \mathrm{e}^{-\mathrm{i} \frac{N}{\mid S_{\mu}(\mathbf{c})} \hat{Q}_{\mu}\left(\mathbf{x}_{i_{\mu \nu}}\right)} \mathrm{d} \mathbf{x}_{i_{\mu \nu}} \\
& =\left\{\prod_{\mu=1}^{K} \prod_{\mathbf{x}} \int \frac{\mathrm{d} \hat{Q}_{\mu}(\mathbf{x})}{2 \pi / N}\right\}
\end{aligned}
$$

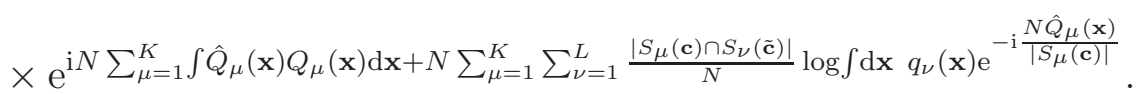

Thus for $P_{N}[Q \mid \boldsymbol{\alpha}(\mathbf{c}, \tilde{\mathbf{c}})] \equiv P_{N}\left[\left\{Q_{\mu}(\mathbf{x})\right\} \mid \mathbf{c}, \tilde{\mathbf{c}}\right]$ we have

$$
P_{N}[Q \mid \boldsymbol{\alpha}(\mathbf{c}, \tilde{\mathbf{c}})]=\int \mathcal{D} \hat{Q} \mathrm{e}^{N \Psi[Q, \hat{Q} \mid \boldsymbol{\alpha}(\mathbf{c}, \tilde{\mathbf{c}})]}
$$

where

$$
\begin{aligned}
\Psi[Q, \hat{Q} \mid \boldsymbol{\alpha}(\mathbf{c}, \tilde{\mathbf{c}})]=\mathrm{i} \sum_{\mu=1}^{K} \int \hat{Q}_{\mu}(\mathbf{x}) Q_{\mu}(\mathbf{x}) \mathrm{d} \mathbf{x} \\
\quad+\sum_{\mu=1}^{K} \sum_{\nu=1}^{L} \alpha(\nu, \mu \mid \mathbf{c}, \tilde{\mathbf{c}}) \log \int q_{\nu}(\mathbf{x}) \mathrm{e}^{\frac{-\mathrm{i}}{\alpha(\mu \mid \mathbf{c})} \hat{Q}_{\mu}(\mathbf{x})} \mathrm{d} \mathbf{x},
\end{aligned}
$$

with the usual short-hand for the path integral measure, $\int \mathcal{D} \hat{Q} \equiv\left\{\prod_{\mu=1}^{K} \prod_{\mathbf{x}} \int\left[\mathrm{d} \hat{Q}_{\mu}(\mathbf{x}) /(2 \pi / N)\right]\right\}$. In the above formula we have also introduced the matrix $\boldsymbol{\alpha}(\mathbf{c}, \tilde{\mathbf{c}})$, with entries $[\boldsymbol{\alpha}(\mathbf{c}, \tilde{\mathbf{c}})]_{\nu \mu}=$ 
$\alpha(\nu, \mu \mid \mathbf{c}, \tilde{\mathbf{c}})$, where in turn $\alpha(\nu, \mu \mid \mathbf{c}, \tilde{\mathbf{c}})=N^{-1}\left|S_{\mu}(\mathbf{c}) \cap S_{\nu}(\tilde{\mathbf{c}})\right|$. We note that $\cup_{\mu=1}^{K}\left(S_{\mu}(\mathbf{c}) \cap S_{\nu}(\tilde{\mathbf{c}})\right)=$ $S_{\nu}(\tilde{\mathbf{c}})$ and that $\cup_{\nu=1}^{L}\left(S_{\mu}(\mathbf{c}) \cap S_{\nu}(\tilde{\mathbf{c}})\right)=S_{\mu}(\mathbf{c})$. From these properties it follows that the entries $\alpha(\nu, \mu \mid \mathbf{c}, \tilde{\mathbf{c}}) \geq 0$ can be interpreted as representing a joint distribution, i.e. $\sum_{\mu=1}^{K} \sum_{\nu=1}^{L} \alpha(\nu, \mu \mid \mathbf{c}, \tilde{\mathbf{c}})=1$, with the marginals $\sum_{\nu=1}^{L} \alpha(\nu, \mu \mid \mathbf{c}, \tilde{\mathbf{c}})=\alpha(\mu \mid \mathbf{c})=\left|S_{\mu}(\mathbf{c})\right| / N$ and $\sum_{\mu=1}^{K} \alpha(\nu, \mu \mid \mathbf{c}, \tilde{\mathbf{c}})=\alpha(\nu \mid \tilde{\mathbf{c}})=\left|S_{\nu}(\tilde{\mathbf{c}})\right| / N$. Using all these ingredients in equation (C12) then leads us to

$$
\begin{aligned}
P_{N}(F) & =\sum_{\mathbf{c}, \tilde{\mathbf{c}}} p(\mathbf{c} \mid K) q(\tilde{\mathbf{c}} \mid L) \int \mathcal{D} Q P_{N}[Q \mid \boldsymbol{\alpha}(\mathbf{c}, \tilde{\mathbf{c}})] \delta\left(F+\sum_{\mu=1}^{K} \frac{\left|S_{\mu}(\mathbf{c})\right|}{N} \int Q_{\mu}(\mathbf{x}) \log p\left(\mathbf{x} \mid \boldsymbol{\theta}_{\mu}\right) \mathrm{d} \mathbf{x}\right) \\
& =\int \mathrm{d} \boldsymbol{\alpha} P_{N}(\boldsymbol{\alpha}) \int \mathcal{D} Q P_{N}[Q \mid \boldsymbol{\alpha}] \delta\left(F+\sum_{\mu=1}^{K} \alpha(\mu) \int Q_{\mu}(\mathbf{x}) \log p\left(\mathbf{x} \mid \boldsymbol{\theta}_{\mu}\right) \mathrm{d} \mathbf{x}\right),
\end{aligned}
$$

where we have defined the integral measure $\int \mathcal{D} Q \equiv\left\{\prod_{\mu=1}^{K} \prod_{\mathbf{x}} \int \mathrm{d} Q_{\mu}(\mathbf{x})\right\}$ as well as the short-hand $\int \mathrm{d} \boldsymbol{\alpha} \equiv \prod_{\mu=1}^{K} \prod_{\nu=1}^{L} \int \mathrm{d} \alpha(\nu, \mu)$. The distribution of $\boldsymbol{\alpha}$ is given by

$$
P_{N}(\boldsymbol{\alpha})=\sum_{\mathbf{c}, \tilde{\mathbf{c}}} p(\mathbf{c} \mid K) q(\tilde{\mathbf{c}} \mid L) \prod_{\mu=1}^{K} \prod_{\nu=1}^{L} \delta[\alpha(\nu, \mu)-\alpha(\nu, \mu \mid \mathbf{c}, \tilde{\mathbf{c}})]
$$

Now for any smooth function $g$ we can consider the following average:

$$
\begin{aligned}
\int P_{N}(F) g(F) \mathrm{d} F & =\int \mathrm{d} \boldsymbol{\alpha} P_{N}(\boldsymbol{\alpha}) \int \mathcal{D} Q P_{N}[Q \mid \boldsymbol{\alpha}] g\left(-\sum_{\mu=1}^{K} \alpha(\mu) \int Q_{\mu}(\mathbf{x}) \log p\left(\mathbf{x} \mid \boldsymbol{\theta}_{\mu}\right) \mathrm{d} \mathbf{x}\right) \\
& =\int \mathrm{d} \boldsymbol{\alpha} P_{N}(\boldsymbol{\alpha}) \frac{\int \mathcal{D} Q P_{N}[Q \mid \boldsymbol{\alpha}]}{\int \mathcal{D} \tilde{Q} P_{N}[\tilde{Q} \mid \boldsymbol{\alpha}]} g\left(-\sum_{\mu=1}^{K} \alpha(\mu) \int Q_{\mu}(\mathbf{x}) \log p\left(\mathbf{x} \mid \boldsymbol{\theta}_{\mu}\right) \mathrm{d} \mathbf{x}\right) \\
& =\int \mathrm{d} \boldsymbol{\alpha} P_{N}(\boldsymbol{\alpha}) \frac{\int \mathcal{D} Q \int \mathcal{D} \hat{Q} \mathrm{e}^{N \Psi[Q, \hat{Q} \mid \boldsymbol{\alpha}]}}{\int \mathcal{D} \tilde{Q} \int \mathcal{D} \hat{Q} \mathrm{e}^{N \Psi[\tilde{Q}, \hat{Q} \mid \boldsymbol{\alpha}]}} \\
& \times g\left(-\sum_{\mu=1}^{K} \alpha(\mu) \int Q_{\mu}(\mathbf{x}) \log p\left(\mathbf{x} \mid \boldsymbol{\theta}_{\mu}\right) \mathrm{d} \mathbf{x}\right) .
\end{aligned}
$$

Let us assume that $P_{N}(\boldsymbol{\alpha}) \rightarrow P(\boldsymbol{\alpha})$ as $N \rightarrow \infty$. Furthermore we expect that in this limit the functional integral in the above equation is dominated by the extremum of the functional $\Psi$ and hence for the distribution $P(F)=\lim _{N \rightarrow \infty} P_{N}(F)$ we obtain

$$
\int P(F) g(F) \mathrm{d} F=\int P(\boldsymbol{\alpha}) g\left(-\sum_{\mu=1}^{K} \alpha(\mu) \int Q_{\mu}(\mathbf{x} \mid \boldsymbol{\alpha}) \log p\left(\mathbf{x} \mid \boldsymbol{\theta}_{\mu}\right) \mathrm{d} \mathbf{x}\right) \mathrm{d} \boldsymbol{\alpha}
$$


where $Q_{\mu}(\mathbf{x} \mid \boldsymbol{\alpha})$ is a solution of the saddle-point equations $\delta \Psi[Q, \hat{Q} \mid \boldsymbol{\alpha}] / \delta Q_{\mu}(\mathbf{x})=0$ and $\delta \Psi[Q, \hat{Q} \mid \boldsymbol{\alpha}] / \delta \hat{Q}_{\mu}(\mathbf{x})=0$. Solving the latter gives us the following two equations:

$$
\begin{aligned}
\mathrm{i} \hat{Q}_{\mu}(\mathbf{x}) & =0 \\
Q_{\mu}(\mathbf{x}) & =\sum_{\nu=1}^{L} \frac{\alpha(\nu, \mu)}{\alpha(\mu)} \frac{q_{\nu}(\mathbf{x}) \mathrm{e}^{\frac{-\mathrm{i}}{\alpha(\mu)} \hat{Q}_{\mu}(\mathbf{x})}}{\int q_{\nu}\left(\mathbf{x}^{\prime}\right) \mathrm{e}^{\frac{-\mathrm{i}}{\alpha(\mu)} \hat{Q}_{\mu}\left(\mathbf{x}^{\prime}\right)} \mathrm{d} \mathbf{x}^{\prime}}
\end{aligned}
$$

from which follows the equation

$$
Q_{\mu}(\mathbf{x} \mid \boldsymbol{\alpha})=\sum_{\nu=1}^{L} \alpha(\nu \mid \mu) q_{\nu}(\mathbf{x})
$$

where $\alpha(\nu \mid \mu)=\alpha(\nu, \mu) / \alpha(\mu)$ is a conditional distribution. From the above we conclude that

$$
\begin{aligned}
P(F)= & \int \mathrm{d} \boldsymbol{\alpha} P(\boldsymbol{\alpha}) \\
& \times \delta\left(F+\sum_{\mu=1}^{K} \sum_{\nu=1}^{L} \alpha(\nu, \mu) \int q_{\nu}(\mathbf{x}) \log p\left(\mathbf{x} \mid \boldsymbol{\theta}_{\mu}\right) \mathrm{d} \mathbf{x}\right) .
\end{aligned}
$$

If we assume that $P(\boldsymbol{\alpha})$ is a delta function, this gives us the mean-field (MF) log-likelihood

$$
F(\boldsymbol{\alpha})=-\sum_{\mu=1}^{K} \sum_{\nu=1}^{L} \alpha(\nu, \mu) \int q_{\nu}(\mathbf{x}) \log p\left(\mathbf{x} \mid \boldsymbol{\theta}_{\mu}\right) \mathrm{d} \mathbf{x}
$$

which is seen to be equivalent to (9). Let us next consider the distribution (C2) of the log-likelihood density (13):

$$
\begin{aligned}
P_{N}(F)= & \sum_{\mathbf{c}} p(\mathbf{c} \mid K) \int \mathrm{d} \mathbf{X} p(\mathbf{X} \mid L) \\
& \times \delta\left(F-\sum_{\mu=1}^{K} \frac{\left|S_{\mu}(\mathbf{c})\right|}{2 N} \log \left((2 \pi \mathrm{e})^{d}\left|\boldsymbol{\Lambda}_{\mu}^{-1}(\mathbf{c}, \mathbf{X})\right|\right)\right)
\end{aligned}
$$

where $\boldsymbol{\Lambda}_{\mu}^{-1}(\mathbf{c}, \mathbf{X})$ is the covariance matrix of the data in cluster $\mu$, which can be written in the form

$$
\mathbf{\Lambda}_{\mu}^{-1}(\mathbf{c}, \mathbf{X})=\frac{1}{\left|S_{\mu}(\mathbf{c})\right|} \sum_{i_{\mu} \in S_{\mu}(\mathbf{c})}\left(\mathbf{x}_{i_{\mu}}-\mathbf{m}_{\mu}(\mathbf{c})\right)\left(\mathbf{x}_{i_{\mu}}-\mathbf{m}_{\mu}(\mathbf{c})\right)^{T}
$$

where $\mathbf{m}_{\mu}(\mathbf{c})=\frac{1}{\left|S_{\mu}(\mathbf{c})\right|} \sum_{i_{\mu} \in S_{\mu}(\mathbf{c})} \mathbf{x}_{i_{\mu}}$. Further manipulation of $P_{N}(F)$ gives

$$
\begin{aligned}
P_{N}(F)=\sum_{\mathbf{c}, \tilde{\mathbf{c}}} p(\mathbf{c} \mid K) & q(\tilde{\mathbf{c}} \mid L) \int\left\{\prod_{\nu=1}^{L} \prod_{i_{\nu} \in S_{\nu}(\tilde{\mathbf{c}})} q_{\nu}\left(\mathbf{x}_{i_{\nu}}\right)\right\} \\
& \times \delta\left(F-\sum_{\mu=1}^{K} \frac{\left|S_{\mu}(\mathbf{c})\right|}{2 N} \log \left((2 \pi \mathrm{e})^{d}\left|\boldsymbol{\Lambda}_{\mu}^{-1}(\mathbf{c}, \mathbf{X})\right|\right)\right) \mathrm{d} \mathbf{X}
\end{aligned}
$$


and the covariance matrix can be written in the form

$$
\begin{aligned}
\Lambda_{\mu}^{-1}(\mathbf{c}, \mathbf{X})= & \frac{1}{\left|S_{\mu}(\mathbf{c})\right|} \sum_{\nu=1}^{L} \sum_{i_{\nu \mu} \in S_{\mu}(\mathbf{c}) \cap S_{\nu}(\tilde{\mathbf{c}})}\left(\mathbf{x}_{i_{\nu \mu}}-\mathbf{m}_{\mu}(\mathbf{c})\right)\left(\mathbf{x}_{i_{\nu \mu}}-\mathbf{m}_{\mu}(\mathbf{c})\right)^{T} \\
=\int \mathrm{d} \mathbf{x} Q_{\mu}(\mathbf{x} \mid \mathbf{c}, \tilde{\mathbf{c}}, \mathbf{X}) & \left(\mathbf{x}-\int Q_{\mu}(\mathbf{y} \mid \mathbf{c}, \tilde{\mathbf{c}}, \mathbf{X}) \mathbf{y} \mathrm{d} \mathbf{y}\right)^{T} \\
& \times\left(\mathbf{x}-\int Q_{\mu}(\mathbf{z} \mid \mathbf{c}, \tilde{\mathbf{c}}, \mathbf{X}) \mathbf{z} \mathrm{d} \mathbf{z}\right)^{T}
\end{aligned}
$$

From the above it is clear that $\hat{F}_{N}$ is a functional of the density $Q_{\mu}(\mathbf{x} \mid \mathbf{c}, \tilde{\mathbf{c}}, \mathbf{X})$, defined in (6) , and the matrix $\boldsymbol{\alpha}(\nu, \mu \mid \mathbf{c} \tilde{\mathbf{c}})$. Following the same steps as in deriving equations (C7)-(C12) gives us

$$
\begin{aligned}
P_{N}(F)= & \int \mathrm{d} \boldsymbol{\alpha} P_{N}(\boldsymbol{\alpha}) \int \mathcal{D} Q P_{N}[Q \mid \boldsymbol{\alpha}] \\
& \times \delta\left(F-\sum_{\mu=1}^{K} \alpha(\mu) \frac{1}{2} \log \left((2 \pi \mathrm{e})^{d}\left|\boldsymbol{\Lambda}_{\mu}^{-1}[Q]\right|\right)\right),
\end{aligned}
$$

where

$$
\begin{aligned}
\boldsymbol{\Lambda}_{\mu}^{-1}[Q]=\int \mathrm{d} \mathbf{x} Q_{\mu}(\mathbf{x})\left(\mathbf{x}-\int\right. & \left.Q_{\mu}(\mathbf{y}) \mathbf{y} \mathrm{d} \mathbf{y}\right) \\
& \times\left(\mathbf{x}-\int Q_{\mu}(\mathbf{z}) \mathbf{z} \mathrm{d} \mathbf{z}\right)^{T} .
\end{aligned}
$$

Furthermore, for $N \rightarrow \infty$, using a similar argument as outlined in equations (C14)-(C19), we obtain

$$
\begin{aligned}
P(F)= & \int \mathrm{d} \boldsymbol{\alpha} P(\boldsymbol{\alpha}) \\
& \times \delta\left(F-\sum_{\mu=1}^{K} \alpha(\mu) \frac{1}{2} \log \left((2 \pi \mathrm{e})^{d}\left|\boldsymbol{\Lambda}_{\mu}^{-1}(\boldsymbol{\alpha})\right|\right)\right)
\end{aligned}
$$

where the covariance matrix $\boldsymbol{\Lambda}_{\mu}^{-1}(\boldsymbol{\alpha})$ is defined by

$$
\boldsymbol{\Lambda}_{\mu}^{-1}(\boldsymbol{\alpha})=\sum_{\nu=1}^{L} \alpha(\nu \mid \mu)\left\langle\left(\mathbf{x}-\mathbf{m}_{\mu}(\boldsymbol{\alpha})\right)\left(\mathbf{x}-\mathbf{m}_{\mu}(\boldsymbol{\alpha})\right)^{T}\right\rangle_{\nu}
$$

where $\mathbf{m}_{\mu}(\boldsymbol{\alpha})=\sum_{\nu=1}^{L} \alpha(\nu \mid \mu)\langle\mathbf{x}\rangle_{\nu}$ is the mean, and we used the short-hand $\langle\{\cdots\}\rangle_{\nu}=$ $\int q_{\nu}(\mathbf{x})\{\cdots\} \mathrm{d} \mathbf{x}$. Assuming that $P(\boldsymbol{\alpha})$ is a delta function subsequently gives us the MF log-likelihood expression (14). 


\section{Appendix D: Proofs of information-theoretic inequalities}

In this section we compute lower bounds for the MF entropy (14). First, we show that $F(\boldsymbol{\alpha})$ satisfies the inequalities

$$
F(\boldsymbol{\alpha}) \geq \sum_{\mu=1}^{K} \alpha(\mu) H\left(Q_{\mu}\right) \geq \sum_{\nu=1}^{L} \gamma(\nu) H\left(q_{\nu}\right)
$$

Let us consider the Kullback-Leibler distance [15] $D\left(Q_{\mu}|| \mathcal{N}_{\mu}\right)$ between the mixture $Q_{\mu}(\mathbf{x})=$ $\sum_{\nu=1}^{L} \alpha(\nu \mid \mu) q_{\nu}(\mathbf{x})$ and the Gaussian distribution $\mathcal{N}\left(\mathbf{x} \mid \mathbf{m}_{\mu}, \boldsymbol{\Lambda}_{\mu}^{-1}\right)$ :

$$
\begin{aligned}
D\left(Q_{\mu}|| \mathcal{N}_{\mu}\right) & =\int \mathrm{d} \mathbf{x} \sum_{\nu=1}^{L} \alpha(\nu \mid \mu) q_{\nu}(\mathbf{x}) \log \left(\frac{\sum_{\nu=1}^{L} \alpha(\nu \mid \mu) q_{\nu}(\mathbf{x})}{\mathcal{N}\left(\mathbf{x} \mid \mathbf{m}_{\mu}, \boldsymbol{\Lambda}_{\mu}^{-1}\right)}\right) \\
& =-H\left(Q_{\mu}\right)-\sum_{\nu=1}^{L} \alpha(\nu \mid \mu) \int \mathrm{d} \mathbf{x} q_{\nu}(\mathbf{x}) \log \mathcal{N}\left(\mathbf{x} \mid \mathbf{m}_{\mu}, \boldsymbol{\Lambda}_{\mu}^{-1}\right) \\
& =-H\left(Q_{\mu}\right)-\sum_{\nu=1}^{L} \alpha(\nu \mid \mu) \int \mathrm{d} \mathbf{x} q_{\nu}(\mathbf{x}) \log \left(\frac{\mathrm{e}^{-\frac{1}{2}\left(\mathbf{x}-\mathbf{m}_{\mu}\right)^{T} \boldsymbol{\Lambda}_{\mu}\left(\mathbf{x}-\mathbf{m}_{\mu}\right)}}{\left|2 \pi \boldsymbol{\Lambda}_{\mu}^{-1}\right|^{\frac{1}{2}}}\right) \\
& =-H\left(Q_{\mu}\right)+\frac{1}{2} \log \left((2 \pi)^{d}\left|\boldsymbol{\Lambda}_{\mu}^{-1}\right|\right) \\
& +\frac{1}{2} \sum_{\nu=1}^{L} \alpha(\nu \mid \mu) \int \mathrm{d} \mathbf{x} q_{\nu}(\mathbf{x})\left(\mathbf{x}-\mathbf{m}_{\mu}\right)^{T} \boldsymbol{\Lambda}_{\mu}\left(\mathbf{x}-\mathbf{m}_{\mu}\right) \\
& =-H\left(Q_{\mu}\right)+\frac{1}{2} \log \left((2 \pi)^{d}\left|\boldsymbol{\Lambda}_{\mu}^{-1}\right|\right) \\
& +\frac{1}{2} \operatorname{Tr}\left\{\boldsymbol{\Lambda}_{\mu} \sum_{\nu=1}^{L} \alpha(\nu \mid \mu) \int q_{\nu}(\mathbf{x})\left(\mathbf{x}-\mathbf{m}_{\mu}\right)\left(\mathbf{x}-\mathbf{m}_{\mu}\right)^{T} \mathrm{~d} \mathbf{x}\right\}
\end{aligned}
$$

Let us define the mean and covariance of the distribution $Q_{\mu}(\mathbf{x})=\sum_{\nu=1}^{L} \alpha(\nu \mid \mu) q_{\nu}(\mathbf{x})$ as $\mathbf{m}_{\mu}=\int Q_{\mu}(\mathbf{x}) \mathbf{x} \mathrm{d} \mathbf{x}$ and $\boldsymbol{\Lambda}_{\mu}^{-1}=\int Q_{\mu}(\mathbf{x})\left(\mathbf{x}-\mathbf{m}_{\mu}\right)\left(\mathbf{x}-\mathbf{m}_{\mu}\right)^{T} \mathrm{~d} \mathbf{x}$. Then $D\left(Q_{\mu}|| \mathcal{N}_{\mu}\right)=-H\left(Q_{\mu}\right)+$ $\frac{1}{2} \log \left((2 \pi \mathrm{e})^{d}\left|\boldsymbol{\Lambda}_{\mu}^{-1}\right|\right)$ and from the simple property $D\left(Q_{\mu}|| \mathcal{N}_{\mu}\right) \geq 0$ we immediately deduce that

$$
F(\boldsymbol{\alpha}) \geq \sum_{\mu=1}^{K} \alpha(\mu) H\left(Q_{\mu}\right)
$$

Furthermore, for the average entropy we find the following inequality 


$$
\begin{aligned}
\sum_{\mu=1}^{K} \alpha(\mu) H\left(Q_{\mu}\right)= & \sum_{\mu=1}^{K} \alpha(\mu) \sum_{\nu_{1}=1}^{L} \alpha\left(\nu_{1} \mid \mu\right) \int q_{\nu_{1}}(\mathbf{x}) \log \left(1 / \sum_{\nu_{2}=1}^{L} \alpha\left(\nu_{2} \mid \mu\right) q_{\nu_{2}}(\mathbf{x})\right) \mathrm{d} \mathbf{x} \\
= & \sum_{\mu=1}^{K} \sum_{\nu_{1}=1}^{L} \alpha\left(\nu_{1}, \mu\right) \\
& \times \int q_{\nu_{1}}(\mathbf{x})\left\{\log q_{\nu_{1}}(\mathbf{x})-\log q_{\nu_{1}}(\mathbf{x})+\log \left(1 / \sum_{\nu_{2}=1}^{L} \alpha\left(\nu_{2} \mid \mu\right) q_{\nu_{2}}(\mathbf{x})\right)\right\} \mathrm{d} \mathbf{x} \\
= & \sum_{\mu=1}^{K} \sum_{\nu=1}^{L} \alpha(\nu, \mu) D\left(q_{\nu}|| Q_{\mu}\right)+\sum_{\nu=1}^{L} \gamma(\nu) H\left(q_{\nu}\right) \\
\geq & \sum_{\nu=1}^{L} \gamma(\nu) H\left(q_{\nu}\right) .
\end{aligned}
$$

Secondly, for the average entropy

$$
F_{0}=\sum_{\nu=1}^{L} \gamma(\nu) \frac{1}{2} \log \left((2 \pi \mathrm{e})^{d}\left|\mathbf{C}_{\nu}\right|\right)
$$

where $\mathbf{C}_{\nu}=\left\langle\mathbf{x} \mathbf{x}^{T}\right\rangle_{\nu}-\langle\mathbf{x}\rangle_{\nu}\langle\mathbf{x}\rangle_{\nu}^{T}$ is the covariance matrix of $q_{\nu}(\mathbf{x})$, we can show that the following holds:

$$
F(\boldsymbol{\alpha}) \geq F_{0}
$$

for all $\boldsymbol{\alpha}$. The above equality follows from properties of the covariance matrix

$$
\begin{aligned}
\boldsymbol{\Lambda}_{\mu}^{-1}(\boldsymbol{\alpha})= & \sum_{\nu=1}^{L} \alpha(\nu \mid \mu) \mathbf{C}_{\nu} \\
& +\sum_{\nu=1}^{L} \alpha(\nu \mid \mu)\left(\langle\mathbf{x}\rangle_{\nu}-\mathbf{m}_{\mu}(\boldsymbol{\alpha})\right)\left(\langle\mathbf{x}\rangle_{\nu}-\mathbf{m}_{\mu}(\boldsymbol{\alpha})\right)^{T}
\end{aligned}
$$

To prove (D5) we first derive the inequality

$$
\log \left|\sum_{\nu=1}^{L} \alpha(\nu) \mathbf{D}_{\nu}\right| \geq \sum_{\nu=1}^{L} \alpha(\nu) \log \left|\mathbf{D}_{\nu}\right|
$$

for symmetric positive definite matrices $\mathbf{D}_{\nu}$ and $\sum_{\nu=1}^{L} \alpha(\nu)=1$, where $\alpha(\nu) \geq 0$. This inequality can be derived by repeated application of Minkowski's inequality for determinants, viz. $|\mathbf{D}+\mathbf{B}|^{\frac{1}{d}} \geq|\mathbf{D}|^{\frac{1}{d}}+|\mathbf{B}|^{\frac{1}{d}}$ for symmetric positive definite matrices $\mathbf{D}$ and $\mathbf{B}$ : 


$$
\begin{aligned}
\left|\sum_{\nu=1}^{L} \alpha(\nu) \mathbf{D}_{\nu}\right|^{\frac{1}{d}}=\mid \alpha(1) \mathbf{D}_{1}+ & \left.\sum_{\nu=2}^{L} \alpha(\nu) \mathbf{D}_{\nu}\right|^{\frac{1}{d}} \\
& \geq \alpha(1)\left|\mathbf{D}_{1}\right|^{\frac{1}{d}}+\left|\sum_{\nu=2}^{L} \alpha(\nu) \mathbf{D}_{\nu}\right|^{\frac{1}{d}} \geq \sum_{\nu=1}^{L} \alpha(\nu)\left|\mathbf{D}_{\nu}\right|^{\frac{1}{d}}
\end{aligned}
$$

from which follows the result

$$
\log \left|\sum_{\nu=1}^{L} \alpha(\nu) \mathbf{D}_{\nu}\right| \geq d \log \left(\sum_{\nu=1}^{L} \alpha(\nu)\left|\mathbf{D}_{\nu}\right|^{\frac{1}{d}}\right) \geq \sum_{\nu=1}^{L} \alpha(\nu) \log \left|\mathbf{D}_{\nu}\right| .
$$

The last step in this argument relied on Jensen's inequality [15]. Let us now apply (D7) to the difference of entropies

$$
\begin{aligned}
& 2\left(F(\boldsymbol{\alpha})-F_{0}\right)=- \sum_{\nu=1}^{L} \gamma(\nu) \log \left|\mathbf{C}_{\nu}\right|+\sum_{\mu=1}^{K} \alpha(\mu) \log \left|\boldsymbol{\Lambda}_{\mu}^{-1}(\boldsymbol{\alpha})\right| \\
&=- \sum_{\nu=1}^{L} \gamma(\nu) \log \left|\mathbf{C}_{\nu}\right|+\sum_{\mu=1}^{K} \alpha(\mu) \\
& \times \log \left|\sum_{\nu=1}^{L} \alpha(\nu \mid \mu)\left(\mathbf{C}_{\nu}+\left(\langle\mathbf{x}\rangle_{\nu}-\mathbf{m}_{\mu}(\boldsymbol{\alpha})\right)\left(\langle\mathbf{x}\rangle_{\nu}-\mathbf{m}_{\mu}(\boldsymbol{\alpha})\right)^{T}\right)\right| \\
& \geq- \sum_{\nu=1}^{L} \gamma(\nu) \log \left|\mathbf{C}_{\nu}\right|+\sum_{\mu=1}^{K} \alpha(\mu) \sum_{\nu=1}^{L} \alpha(\nu \mid \mu) \\
& \quad \times \log \left|\mathbf{C}_{\nu}+\left(\langle\mathbf{x}\rangle_{\nu}-\mathbf{m}_{\mu}(\boldsymbol{\alpha})\right)\left(\langle\mathbf{x}\rangle_{\nu}-\mathbf{m}_{\mu}(\boldsymbol{\alpha})\right)^{T}\right| \\
& \geq-\sum_{\nu=1}^{L} \gamma(\nu) \log \left|\mathbf{C}_{\nu}\right|+d \sum_{\mu=1}^{K} \alpha(\mu) \sum_{\nu=1}^{L} \alpha(\nu \mid \mu) \\
& \quad \times \log \left(\left|\mathbf{C}_{\nu}\right|^{\frac{1}{d}}+\left|\left(\langle\mathbf{x}\rangle_{\nu}-\mathbf{m}_{\mu}(\boldsymbol{\alpha})\right)\left(\langle\mathbf{x}\rangle_{\nu}-\mathbf{m}_{\mu}(\boldsymbol{\alpha})\right)^{T}\right|^{\frac{1}{d}}\right) .
\end{aligned}
$$

The last line in the above, obtained by Minkowski's inequality, is equal to zero, and hence $F(\boldsymbol{\alpha}) \geq F_{0}$ for all $\boldsymbol{\alpha}$.

\section{Appendix E: Algorithmic cost of ordering random unbiased partitions}

Let us assume that we have $N$ 'particles' of $L$ different 'colours' which are distributed into $K$ different reservoirs. The probability that a particle has colour $\nu \in[L]$ is $\gamma(\nu)$ and that it is in the reservoir $\mu$ is $1 / K$. Assuming that colour and reservoir allocation are independent events, the probability of 'configuration' $\mathbf{A}=\left(\mathbf{a}_{1}, \ldots, \mathbf{a}_{N}\right)$, where $\mathbf{a}_{i}=\left(a_{i}(1), a_{i}(2)\right)$ with 
the colour $a_{i}(1) \in[L]$ and reservoir number $a_{i}(2) \in[K]$ of the particle $i$, is given by

$$
\begin{aligned}
& P(\mathbf{A})=\prod_{i=1}^{N} P\left(\mathbf{a}_{i}\right), \\
& P\left(\mathbf{a}_{i}\right) \equiv P\left(a_{i}(1)=\nu, a_{i}(2)=\mu\right)=\frac{\gamma(\nu)}{K} .
\end{aligned}
$$

The total number of particles in reservoir $\mu$ is given by $N_{\mu}(\mathbf{A})=\sum_{i=1}^{N} \delta_{\mu ; a_{i}(2)}$. Let us now consider the joint distribution of particle numbers in reservoirs

$$
\begin{aligned}
P\left(N_{1}, \ldots, N_{K}\right) & =\sum_{\mathbf{A}} P(\mathbf{A}) \prod_{\mu=1}^{K} \delta_{N_{\mu} ; N_{\mu}(\mathbf{A})} \\
& =K^{-N} \sum_{a_{1}(2), \ldots, a_{N}(2)} \prod_{\mu=1}^{K} \delta_{N_{\mu} ; \sum_{i=1}^{N} \delta_{\mu ; a_{i}(2)}} \\
& =K^{-N} \frac{N !}{\prod_{\mu=1}^{K} N_{\mu} !},
\end{aligned}
$$

where $\sum_{\mu=1}^{K} N_{\mu}=N$. The probability of observing the event that at least one reservoir is empty is given by

$$
\begin{aligned}
1- & P\left(N_{1}>0, \ldots, N_{K}>0\right) \\
& =1-\sum_{N_{1}>0, \ldots, N_{K}>0} K^{-N} \frac{N !}{\prod_{\mu=1}^{K} N_{\mu} !} \\
& =K^{-N}\left(\sum_{N_{1} \geq 0, \ldots, N_{K} \geq 0} \frac{N !}{\prod_{\mu=1}^{K} N_{\mu} !}\right. \\
& \left.-\sum_{N_{1}>0, \ldots, N_{K}>0} \frac{N !}{\prod_{\mu=1}^{K} N_{\mu} !}\right) \\
& =\sum_{\ell=1}^{K-1}\left(\begin{array}{c}
K \\
\ell
\end{array}\right)\left(1-\frac{\ell}{K}\right)^{N} .
\end{aligned}
$$

Thus the probability of this event decays exponentially with increasing $N$ and, as $N \rightarrow$ $\infty$, the sequence $a_{1}(2), \ldots, a_{N}(2)$, sampled from the distribution (E2) is, with high probability, a partition of the set $[N]$ into $K$ subsets (or clusters). Furthermore, the entropy density $N^{-1} \log \left(K^{N}\right)=\log K$ of such sequences approaches the entropy density $N^{-1} \log (K ! \mathcal{S}(N, K))$ of the random partitions sampled uniformly from (A13).

Let us assume that $K \leq L$. The total number of particles of colour $\nu$, and the number of particles of colour $\nu$ in reservoir $\mu$ are given, respectively, by $N_{\nu}(\mathbf{A})=\sum_{i=1}^{N} \delta_{\nu ; a_{i}(1)}$ and $N_{\nu \mu}(\mathbf{A})=\sum_{i=1}^{N} \delta_{\nu ; a_{i}(1)} \delta_{\mu ; a_{i}(2)}$. The number of particles of colour $\nu$ which are not in 
reservoir $\mu$ is the difference $N_{\nu}(\mathbf{A})-N_{\nu \mu}(\mathbf{A})$. Suppose that each reservoir has a preference for particles of a particular colour (or colours), i.e. there is an onto mapping $\nu \rightarrow \mu(\nu)$ between colours and reservoirs, then the total number of particles which are not in 'their' reservoirs, i.e. the number of particles which are to be 'moved' in order for all particles to be in reservoirs to which they belong, is given by the difference $\sum_{\nu=1}^{L}\left(N_{\nu}(\mathbf{A})-N_{\nu \mu(\nu)}(\mathbf{A})\right)=$ $N-\sum_{\nu=1}^{L} N_{\nu \mu(\nu)}(\mathbf{A})$.

We are interested in the average and variance of $N-\sum_{\nu=1}^{L} N_{\nu \mu(\nu)}(\mathbf{A})$. The average is given by

$$
\begin{aligned}
\left\langle N-\sum_{\nu=1}^{L} N_{\nu \mu(\nu)}(\mathbf{A})\right\rangle_{\mathbf{A}} & =N-\sum_{\nu=1}^{L} \sum_{i=1}^{N}\left\langle\delta_{\nu ; a_{i}(1)} \delta_{\mu(\nu) ; a_{i}(2)}\right\rangle_{\mathbf{A}} \\
& =N-\sum_{\nu=1}^{L} \sum_{i=1}^{N} \frac{\gamma(\nu)}{K} \\
& =N \frac{K-1}{K}
\end{aligned}
$$

and the variance is given by

$$
\begin{aligned}
\operatorname{Var}\left\{N-\sum_{\nu=1}^{L} N_{\nu \mu(\nu)}(\mathbf{A})\right\} & =\operatorname{Var}\left\{\sum_{\nu=1}^{L} N_{\nu \mu(\nu)}(\mathbf{A})\right\} \\
& =\left\langle\left(\sum_{\nu=1}^{L} N_{\nu \mu(\nu)}(\mathbf{A})-\frac{N}{K}\right)^{2}\right\rangle_{\mathbf{A}} \\
& =\frac{N}{K}\left(1-\frac{1}{K}\right) .
\end{aligned}
$$

The average in the penultimate line of the above was computed as follows

$$
\begin{aligned}
\langle & \left.\left(\sum_{\nu=1}^{L} N_{\nu \mu(\nu)}(\mathbf{A})\right)^{2}\right\rangle_{\mathbf{A}} \\
= & \sum_{\nu} \sum_{i_{1}, i_{2}}\left\langle\delta_{\nu ; a_{i_{1}}(1)} \delta_{\mu(\nu) ; a_{i_{1}}(2)} \delta_{\nu ; a_{i_{2}}(1)} \delta_{\mu(\nu) ; a_{i_{2}}(2)}\right\rangle_{\mathbf{A}}+ \\
& \sum_{\nu_{1} \neq \nu_{2}} \sum_{i_{1}, i_{2}=1}\left\langle\delta_{\nu_{1} ; a_{i_{1}}(1)} \delta_{\mu\left(\nu_{1}\right) ; a_{i_{1}}(2)} \delta_{\nu_{2} ; a_{i_{2}}(1)} \delta_{\mu\left(\nu_{2}\right) ; a_{i_{2}}(2)}\right\rangle_{\mathbf{A}} \\
= & \frac{N}{K}+\frac{N(N-1)}{K^{2}} \sum_{\nu=1}^{L} \gamma^{2}(\nu)+\frac{N(N-1)}{K^{2}} \sum_{\nu_{1} \neq \nu_{2}} \gamma\left(\nu_{1}\right) \gamma\left(\nu_{2}\right) \\
= & \frac{N}{K}+\frac{N(N-1)}{K^{2}} .
\end{aligned}
$$

From the above derivations it follows that for a random unbiased partition to be ordered, i.e. for particles of the same colour to occupy at most one reservoir, a fraction of particles 

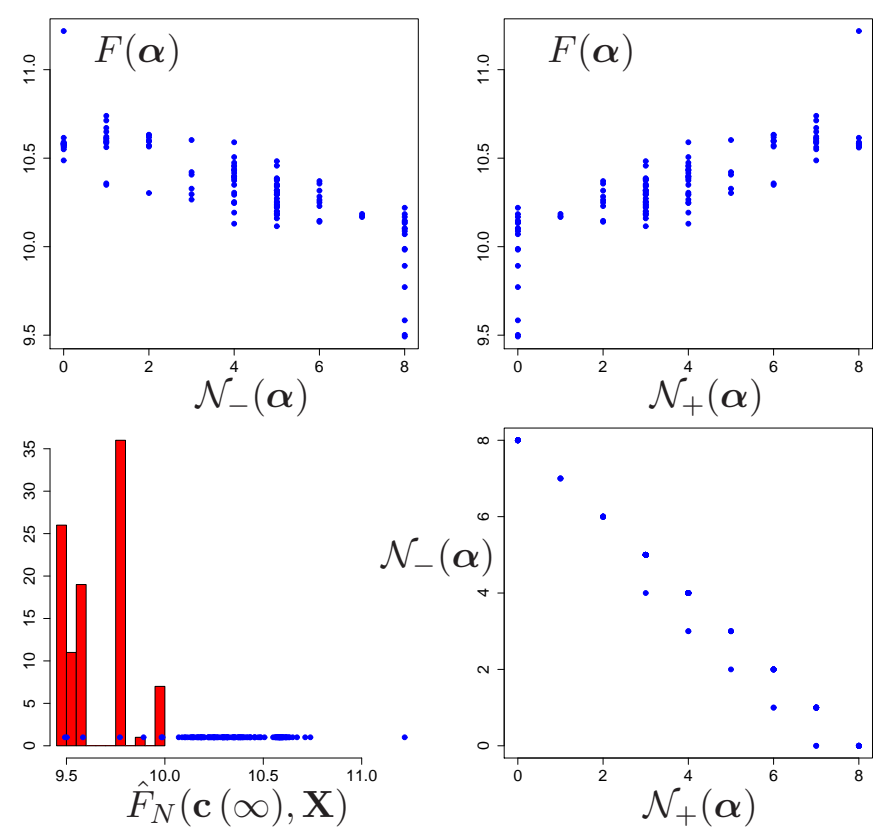

FIG. 6. (Color online) Top left: $F(\boldsymbol{\alpha})$ as a function of the number of $F$-increasing directions $\mathcal{N}_{-}(\boldsymbol{\alpha})$. Top: right: $F(\boldsymbol{\alpha})$ as a function of the number of $F$-decreasing directions $\mathcal{N}_{+}(\boldsymbol{\alpha})$. Bottom left: histogram of log-likelihood values $\hat{F}_{N}(\mathbf{c}(\infty), \mathbf{X})$, obtained by running gradient descent from a 100 different random unbiased partitions, with the assumed number $K=2$ of clusters. Blue filled circles correspond to the MF log-likelihood, $F(\boldsymbol{\alpha})$, computed for all possible values of $\alpha(\nu, \mu)=$ $\mathbb{1}\left[\nu \in S_{\mu}\right] \gamma(\nu)$. Bottom right: $\mathcal{N}_{-}(\boldsymbol{\alpha})$ as a function of $\mathcal{N}_{+}(\boldsymbol{\alpha})$.

has to be moved that is on average $\left\langle 1-\frac{1}{N} \sum_{\nu=1}^{L} N_{\nu \mu(\nu)}(\mathbf{A})\right\rangle_{\mathbf{A}}=(K-1) / K$, with variance $\operatorname{Var}\left\{1-\frac{1}{N} \sum_{\nu=1}^{L} N_{\nu \mu(\nu)}(\mathbf{A})\right\}=\left(1-K^{-1}\right) / N K$.

\section{Appendix F: Details of numerical experiments}

In this section we study the performance of the simplest algorithm that minimises the log-likelihood function (13) via gradient descent, for the data described in Figure 2. The algorithm is implemented as follows:

1. Start with any initial partition $\Pi(\mathbf{c}(0))=\left\{S_{1}(\mathbf{c}(0)), \ldots, S_{K}(\mathbf{c}(0))\right\}$, and compute the log-likelihood $\hat{F}_{N}(\mathbf{c}(0), \mathbf{X})$.

2. For all $i \in[N]$, consider all possible moves of $i$ from its current cluster $S_{\mu}(\mathbf{c})$ to a new cluster $S_{\nu}(\mathbf{c})$ and compute the new value $\hat{F}_{N}(\mathbf{c}, \mathbf{X})$ for each. 

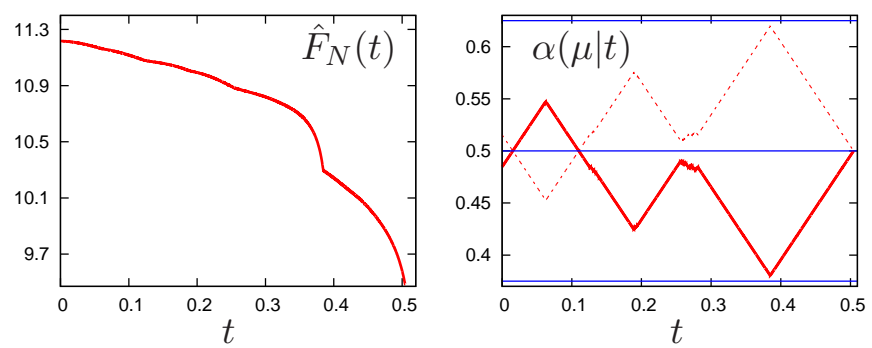

FIG. 7. (Color online) Evolution of the log-likelihood, $\hat{F}_{N}(t) \equiv \hat{F}_{N}(\mathbf{c}(t), \mathbf{X})$, and the fraction of data in cluster $\mu, \alpha(\mu \mid t) \equiv \alpha(\mu \mid \mathbf{c}(t))$, where $\mu=\{1,2\}$, shown as functions of time (normalised number of 'moves') in the gradient descent algorithm evolving from a random unbiased initial partition. The assumed number of clusters is $K=2$. Blue horizontal lines correspond to the levels $3 / 8,4 / 8$ and $5 / 8$.

3. Select and execute a move which gives the largest decrease in $\hat{F}_{N}(\mathbf{c}, \mathbf{X})$, and update $\Pi(\mathbf{c})$.

4. Continue the last two steps while the value of $\hat{F}_{N}(\mathbf{c}, \mathbf{X})$ continues to change.

5. Output the partition $\Pi(\mathbf{c}(\infty))$ and the value of $\hat{F}_{N}(\mathbf{c}(\infty), \mathbf{X})$.

Using as initial states random partitions of data $\mathbf{c}(0)$, where each $i \in[N]$ has a probability $1 / K$ of being allocated to one of the $K$ clusters2, we run the above algorithm for each value of $K \in[17]$ for 100 different initalisations $\mathbf{c}(0)$ and select the final partition, $\mathbf{c}(\infty)$, with the smallest value of $\hat{F}_{N} \equiv \hat{F}_{N}(\mathbf{c}(\infty), \mathbf{X})$. The latter is our estimate of $\min _{\mathbf{c}} \hat{F}_{N}(\mathbf{c}, \mathbf{X})$. We also compute, with the same parameters used to generate our data, the mean-field log-likelihood $F(\boldsymbol{\alpha})$ via equation (14).

When $K \leq L$, the log-likelihood $\hat{F}_{N}(\mathbf{c}(\infty), \mathbf{X})$ is dominated by partitions $\mathbf{c}(\infty)$ corresponding to local minima and saddlepoints of $F(\boldsymbol{\alpha})$. The matrix $\boldsymbol{\alpha}$ is defined by the entries $[\boldsymbol{\alpha}]_{\nu \mu}=\mathbb{1}\left[\nu \in S_{\mu}\right] \gamma(\nu)$, generated by partitions $\Pi=\left\{S_{1}, \ldots, S_{K}\right\}$ of the set $[L]$ into $K$ subsets. The total number of partitions is given by $\mathcal{S}(L, K)$. To enumerate all partitions we use the algorithm of [25]. We classify turning points of $F(\boldsymbol{\alpha})$ as follows. For a given $\Pi$ and its associated matrix $\boldsymbol{\alpha}$ we count the number $\mathcal{N}_{+}(\boldsymbol{\alpha})$ of elementary 'moves' into the new partition $\tilde{\Pi}$ and $\tilde{\boldsymbol{\alpha}}$ (in a single elementary 'move', a member of the set $S_{\mu}$, with $\left|S_{\mu}\right|>1$, is

\footnotetext{
${ }^{2}$ In section $\mathrm{E}$ we proved that for $N \rightarrow \infty$ the matrix c constructed in this way is, with high probability, a partition of the set $[N]$ into the $K$ subsets.
} 

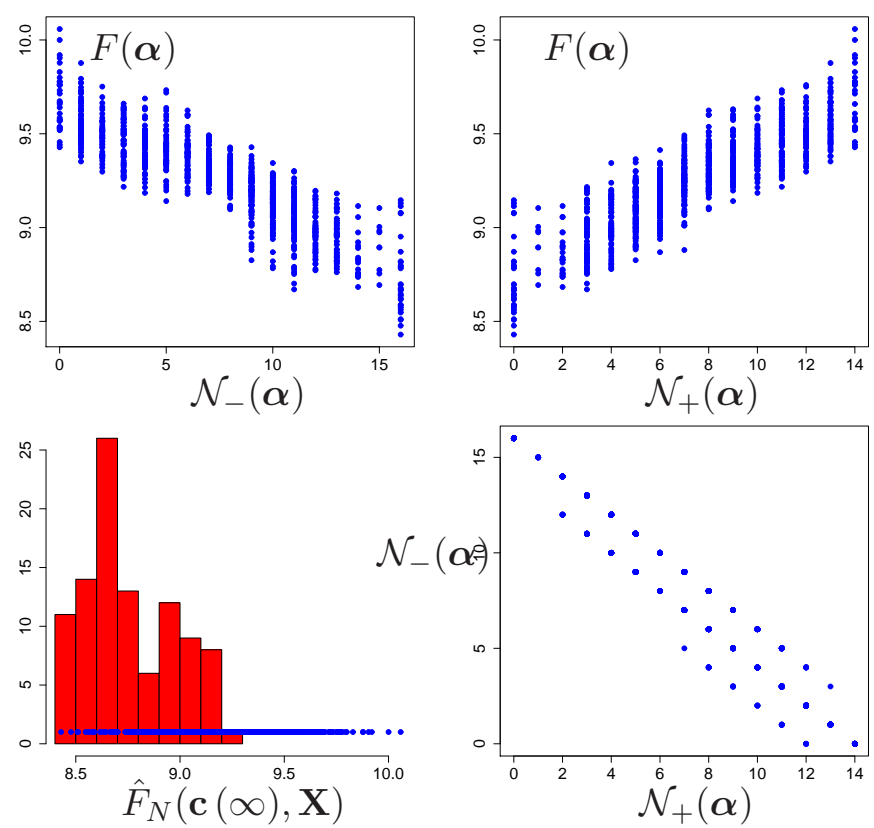

FIG. 8. (Color online) Top left: $F(\boldsymbol{\alpha})$ as a function of the number of $F$-increasing directions $\mathcal{N}_{-}(\boldsymbol{\alpha})$. Top: right: $F(\boldsymbol{\alpha})$ as a function of the number of $F$-decreasing directions $\mathcal{N}_{+}(\boldsymbol{\alpha})$. Bottom left: histogram of log-likelihood values $\hat{F}_{N}(\mathbf{c}(\infty), \mathbf{X})$, obtained by running gradient descent from a 100 different random unbiased partitions, with the assumed number $K=3$ of clusters. Blue filled circles correspond to the MF log-likelihood, $F(\boldsymbol{\alpha})$, computed for all possible values of $\alpha(\nu, \mu)=$ $\mathbb{1}\left[\nu \in S_{\mu}\right] \gamma(\nu)$. Bottom right: $\mathcal{N}_{-}(\boldsymbol{\alpha})$ as a function of $\mathcal{N}_{+}(\boldsymbol{\alpha})$.

moved into the set $\left.S_{\nu}\right)$ for which $F(\boldsymbol{\alpha})>F(\tilde{\boldsymbol{\alpha}})$, and the number $\mathcal{N}_{-}(\boldsymbol{\alpha})$ of moves for which $F(\boldsymbol{\alpha})<F(\tilde{\boldsymbol{\alpha}})$. If $\mathcal{N}_{+}(\boldsymbol{\alpha})=0$ the state $\boldsymbol{\alpha}$ is a (possibly local) minimum, and if $\mathcal{N}_{-}(\boldsymbol{\alpha})=0$ the state $\boldsymbol{\alpha}$ is a (possibly local) maximum. All other cases are saddle points. In Figures 6, 8, 10 and 12 we compare $\hat{F}_{N}(\mathbf{c}(\infty), \mathbf{X})$ with $F(\boldsymbol{\alpha})$.

Those turning points of $F(\boldsymbol{\alpha})$ that are of the form $[\boldsymbol{\alpha}]_{\nu \mu}=\mathbb{1}\left[\nu \in S_{\mu}\right] \gamma(\nu)$ also act as dynamic 'attractors'. This can be seen by comparing Figure 6 to Figure 7, and Figure 8 to Figure 9, etc. Here $\hat{F}_{N}(t) \equiv \hat{F}_{N}(\mathbf{c}(t), \mathbf{X})$, as computed during the simulated process, is seen to evolve from plateau to plateau by a succession of rapid relaxations, and the value of $\hat{F}_{N}(t)$ at the beginning of each plateau can be (approximately) mapped to the value of $F(\boldsymbol{\alpha})$ via the fractions $\alpha(\mu)=\sum_{\nu=1}^{L} \mathbb{1}\left[\nu \in S_{\mu}\right] \gamma(\nu)$ of data in clusters $\mu$. However as $K$ is increased, more and more attractors are not of the form $\mathbb{1}\left[\nu \in S_{\mu}\right] \gamma(\nu)$ (see Figures 9, 11] and 131$)$.

The predictions of the mean-field $\log$-likelihood $F(\boldsymbol{\alpha})$ for $\min _{\mathbf{c}} \hat{F}_{N}(\mathbf{c}, \mathbf{X})$ are incorrect 

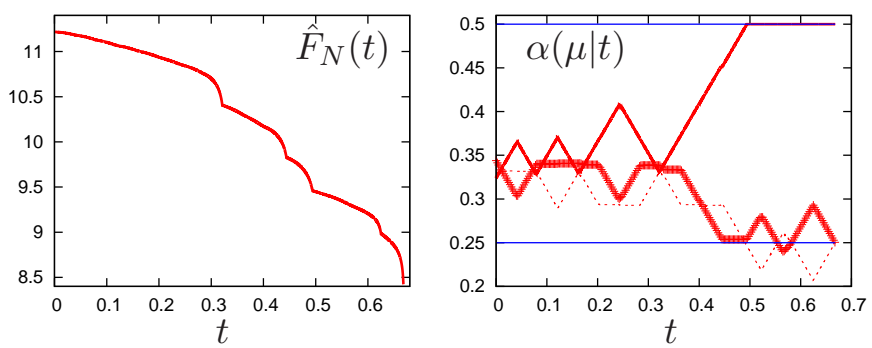

FIG. 9. (Color online) Evolution of the log-likelihood, $\hat{F}_{N}(t) \equiv \hat{F}_{N}(\mathbf{c}(t), \mathbf{X})$, and the fraction of data in cluster $\mu, \alpha(\mu \mid t) \equiv \alpha(\mu \mid \mathbf{c}(t))$, where $\mu=\{1,2,3\}$, shown as functions of time (normalised number of 'moves') in the gradient descent algorithm evolving from a random unbiased initial partition. The assumed number of clusters is $K=3$. Blue horizontal lines correspond to the levels $3 / 8,4 / 8$ and $5 / 8$.

when $K>L$. The log-likelihood $F(\boldsymbol{\alpha})$ is bounded from below by the average entropy $\sum_{\nu=1}^{L} \gamma(\nu) H\left(q_{\nu}\right)$, but in this regime the gap between this lower bound and $\min _{\mathbf{c}} \hat{F}_{N}(\mathbf{c}, \mathbf{X})$ is widening as we increase the number of assumed clusters $K$. This effect can be clearly seen in Figure 14. We also see in this Figure that $\sum_{\nu=1}^{L} \gamma(\nu) H\left(q_{\nu}\right)$ separates the low entropy states obtained by gradient descent into two sets. The first set, which includes $\operatorname{argmin}_{\mathbf{c}} \hat{F}_{N}(\mathbf{c}, \mathbf{X})$, is given by $3\left\{\mathbf{c}: \hat{F}_{N}(\mathbf{c}, \mathbf{X}) \leq \sum_{\nu=1}^{L} \gamma(\nu) H\left(q_{\nu}\right)\right\}$, and the second set is given by $\left\{\mathbf{c}: \hat{F}_{N}(\mathbf{c}, \mathbf{X})>\right.$ $\left.\sum_{\nu=1}^{L} \gamma(\nu) H\left(q_{\nu}\right)\right\}$. Since for $K>L$ we have $F(\boldsymbol{\alpha})>\sum_{\nu=1}^{L} \gamma(\nu) H\left(q_{\nu}\right)$, we expect that $\min _{\boldsymbol{\alpha}} F(\boldsymbol{\alpha})$ gives correct predictions for at least some of the low entropy states in the second set.

${ }^{3}$ The equality in this definition can only be true when $K=L$ (see Figure 12). 

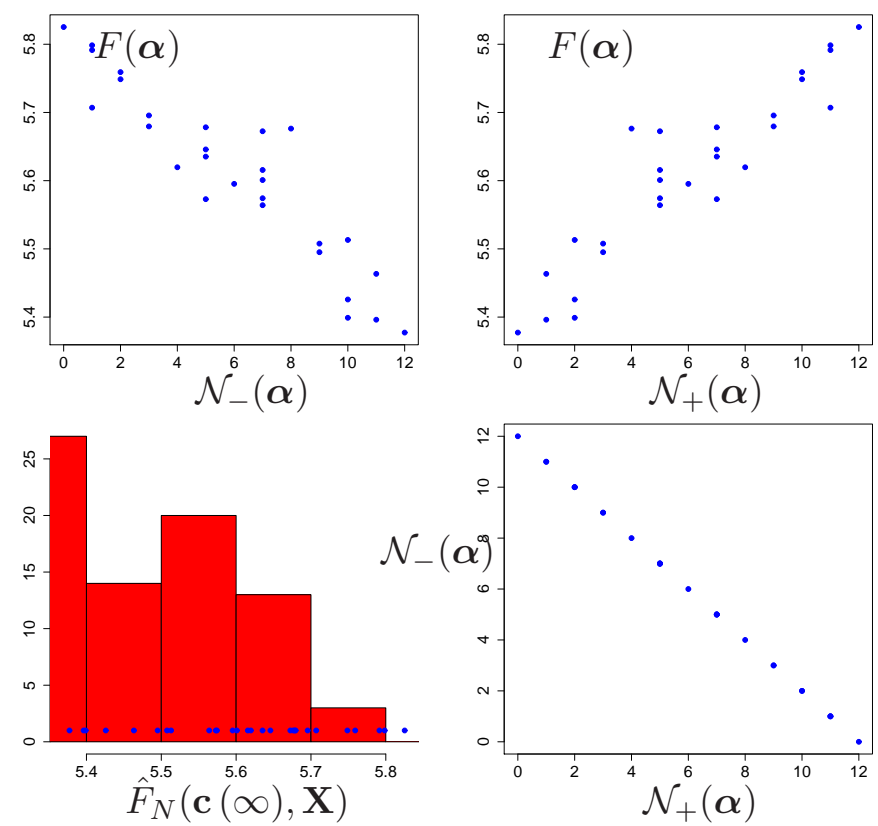

FIG. 10. (Color online) Top left: $F(\boldsymbol{\alpha})$ as a function of the number of $F$-increasing directions $\mathcal{N}_{-}(\boldsymbol{\alpha})$. Top: right: $F(\boldsymbol{\alpha})$ as a function of the number of $F$-decreasing directions $\mathcal{N}_{+}(\boldsymbol{\alpha})$. Bottom left: histogram of log-likelihood values $\hat{F}_{N}(\mathbf{c}(\infty), \mathbf{X})$, obtained by running gradient descent from a 100 different random unbiased partitions, with the assumed number $K=7$ of clusters. Blue filled circles correspond to the MF log-likelihood, $F(\boldsymbol{\alpha})$, computed for all possible values of $\alpha(\nu, \mu)=$ $\mathbb{1}\left[\nu \in S_{\mu}\right] \gamma(\nu)$. Bottom right: $\mathcal{N}_{-}(\boldsymbol{\alpha})$ as a function of $\mathcal{N}_{+}(\boldsymbol{\alpha})$.
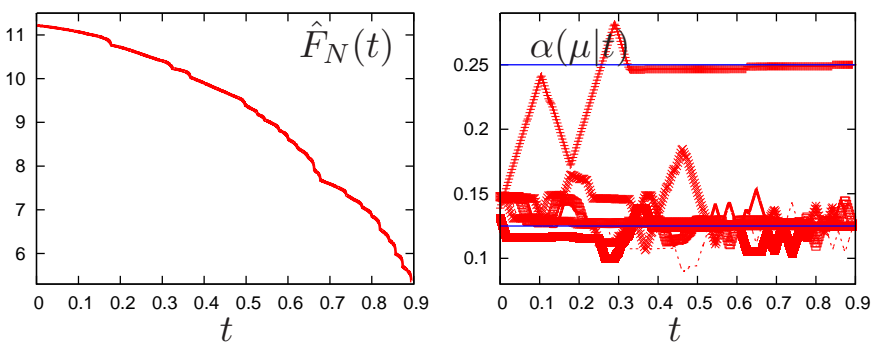

FIG. 11. (Color online) Evolution of the log-likelihood, $\hat{F}_{N}(t) \equiv \hat{F}_{N}(\mathbf{c}(t), \mathbf{X})$, and the fraction of data in cluster $\mu, \alpha(\mu \mid t) \equiv \alpha(\mu \mid \mathbf{c}(t))$, where $\mu=\{1,2, \ldots, 7\}$, shown as functions of time (normalised number of 'moves') in the gradient descent algorithm evolving from a random unbiased initial partition. The assumed number of clusters is $K=7$. Blue horizontal lines correspond to the levels $3 / 8,4 / 8$ and $5 / 8$. 


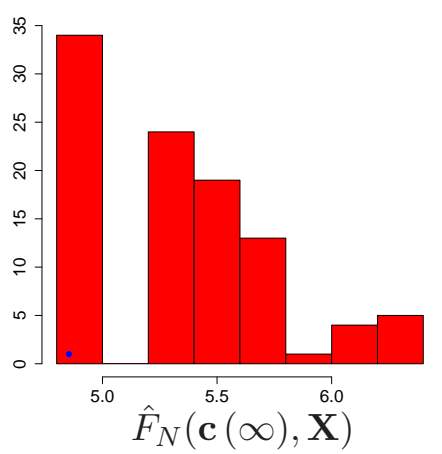

FIG. 12. (Color online) Histogram of the log-likelihood values obtained by running the gradient descent algorithm from a 100 different random unbiased partitions, with the assumed number $K=8$ of clusters. The blue filled circle corresponds to the MF lower bound $\sum_{\nu=1}^{L} \gamma(\nu) H\left(q_{\nu}\right)=4.853905$.
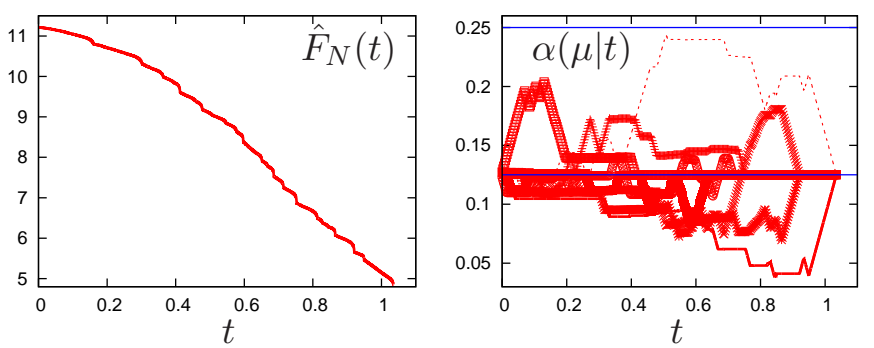

FIG. 13. (Color online) Evolution of the log-likelihood, $\hat{F}_{N}(t) \equiv \hat{F}_{N}(\mathbf{c}(t), \mathbf{X})$, and the fraction of data in cluster $\mu, \alpha(\mu \mid t) \equiv \alpha(\mu \mid \mathbf{c}(t))$, where $\mu=\{1,2, \ldots, 8\}$, shown as functions of time (normalised number of 'moves') in the gradient descent algorithm evolving from a random unbiased initial partition. The assumed number of clusters is $K=8$. Blue horizontal lines correspond to the levels $3 / 8,4 / 8$ and $5 / 8$. 


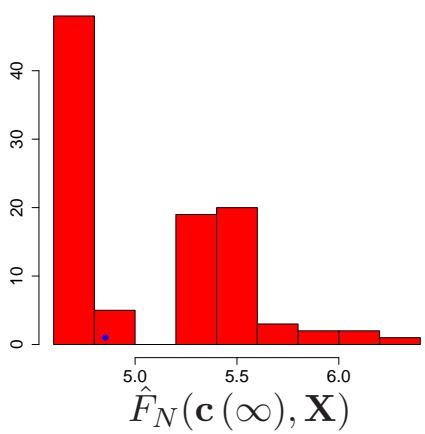

FIG. 14. (Color online) Histogram of the log-likelihood values obtained by running the gradient descent algorithm from a 100 different random unbiased partitions, with the assumed number $K=9$ of clusters. The blue filled circle corresponds to the MF lower bound $\sum_{\nu=1}^{L} \gamma(\nu) H\left(q_{\nu}\right)=4.853905$. 

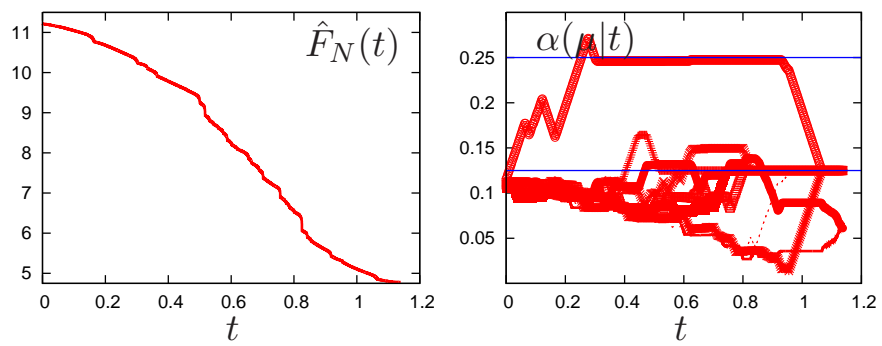

FIG. 15. (Color online) Evolution of the log-likelihood, $\hat{F}_{N}(t) \equiv \hat{F}_{N}(\mathbf{c}(t), \mathbf{X})$, and the fraction of data in cluster $\mu, \alpha(\mu \mid t) \equiv \alpha(\mu \mid \mathbf{c}(t))$, where $\mu=\{1,2, \ldots, 9\}$, shown as functions of time (normalised number of 'moves') in the gradient descent algorithm evolving from a random unbiased initial partition. The assumed number of clusters is $K=9$. Blue horizontal lines correspond to the levels $3 / 8,4 / 8$ and $5 / 8$.

\section{Appendix G: Estimation of differential entropy}

In this section we compute the finite sample-size corrections to the MF entropy (14). In order to do this we first note that for a sample $\left\{\mathbf{x}_{1}, \ldots, \mathbf{x}_{N}\right\}$, where each $\mathbf{x}_{i} \in \mathbb{R}^{d}$ is drawn from the multivariate Gaussian distribution $\mathcal{N}(\mathbf{x} \mid \mathbf{m}, \boldsymbol{\Lambda})$, the empirical covariance matrix $\hat{\mathbf{\Lambda}}=N^{-1} \sum_{i=1}^{N}\left(\mathbf{x}_{i}-\hat{\mathbf{m}}\right)\left(\mathbf{x}_{i}-\hat{\mathbf{m}}\right)^{T}$, where $\hat{\mathbf{m}}=\frac{1}{N} \sum_{i=1}^{N} \mathbf{x}_{i}$ is the empirical mean, obeys the following asymptotic law: $[\log |\hat{\boldsymbol{\Lambda}}|-\log |\boldsymbol{\Lambda}|-d(d+1) / 2 N] / \sqrt{2 d / N} \rightarrow \mathcal{N}(0,1)$ as $N \rightarrow \infty$ (see [19] and references therein). This is equivalent to stating $\log |\hat{\boldsymbol{\Lambda}}| \rightarrow \log |\boldsymbol{\Lambda}|+d(d+$ 1) $/ 2 N+z \sqrt{2 d / N}$, where $z \sim \mathcal{N}(0,1)$.

Let us assume that the above is true for the empirical covariance matrices that feature 
in the log-likelihood (13) and evaluate $\hat{F}_{N}(\mathbf{c})$ for large $N$ :

$$
\begin{aligned}
\hat{F}_{N}(\mathbf{c})= & \sum_{\mu=1}^{K} \frac{M_{\mu}(\mathbf{c})}{N} \frac{1}{2} \log \left((2 \pi \mathrm{e})^{d}\left|\boldsymbol{\Lambda}_{\mu}^{-1}(\mathbf{c})\right|\right) \\
= & \sum_{\mu=1}^{K} \frac{M_{\mu}(\mathbf{c})}{2 N}\left\{\log \left((2 \pi \mathrm{e})^{d}\left|\boldsymbol{\Lambda}_{\mu}^{-1}(\boldsymbol{\alpha})\right|\right)\right. \\
& \left.+\frac{d(d+1)}{2 M_{\mu}(\mathbf{c})}+z_{\mu} \sqrt{\frac{2 d}{M_{\mu}(\mathbf{c})}}\right\} \\
= & F(\boldsymbol{\alpha})+\sum_{\mu=1}^{K} \frac{M_{\mu}(\mathbf{c})}{2 N}\left\{\frac{d(d+1)}{2 M_{\mu}(\mathbf{c})}+z_{\mu} \sqrt{\frac{2 d}{M_{\mu}(\mathbf{c})}}\right\} \\
= & F(\boldsymbol{\alpha})+\frac{K d(d+1)}{4 N}+\sum_{\mu=1}^{K} z_{\mu} \sqrt{\frac{d \alpha(\mu)}{2 N}}
\end{aligned}
$$

The average and variance of the above random variable are given by $F(\boldsymbol{\alpha})+K d(d+1) / 4 N$ and $d / 2 N$, respectively. We expect the above result to be exact when $F(\boldsymbol{\alpha})=\sum_{\nu=1}^{L} \gamma(\nu) H\left(q_{\nu}\right)$, which can only happen when $K=L$, and all $q_{\nu}(\mathbf{x})$ are Gaussian distributions.

[1] M. A. Kuhn, E. D. Feigelson, K. V. Getman, A. J. Baddeley, P. S. Broos, A. Sills, M. R. Bate, M. S. Povich, K. L. Luhman, H. A. Busk, T. Naylor, and R. R. King, Astrophys. J. 787, 107 (2014).

[2] R. de Souza, M. Dantas, M. Costa-Duarte, E. Feigelson, M. Killedar, P.-Y. Lablanche, R. Vilalta, A. Krone-Martins, R. Beck, and F. Gieseke, arXiv preprint arXiv:1703.07607 (2017).

[3] W. P. Hanage, C. Fraser, J. Tang, T. R. Connor, and J. Corander, Science 324, 1454 (2009).

[4] V. G. Martin, Y.-C. B. Wu, C. L. Townsend, G. H. C. Lu, J. S. OHare, A. Mozeika, A. C. C. Coolen, D. Kipling, F. Fraternali, and D. K. Dunn-Walters, Frontiers in Immunology 7, 546 (2016)

[5] C. M. Bishop, Pattern recognition and machine learning (Springer, 2006).

[6] B. J. Frey and D. Dueck, Science 315, 972 (2007).

[7] A. Rodriguez and A. Laio, Science 344, 1492 (2014).

[8] C. Fraley and A. E. Raftery, J. Am. Stat. Assoc. 97, 611 (2002).

[9] A. Nobile and A. T. Fearnside, Stat. Comput. 17, 147 (2007).

[10] J. Corander, M. Gyllenberg, and T. Koski, Adv. Data Anal. Classi. 3, 3 (2009). 
[11] M. Mézard and A. Montanari, Information, physics, and computation (Oxford University Press, 2009).

[12] K. Rose, E. Gurewitz, and G. C. Fox, Phys. Rev. Lett. 65, 945 (1990).

[13] M. Blatt, S. Wiseman, and E. Domany, Phys. Rev. Lett. 76, 3251 (1996).

[14] M. Mézard, G. Parisi, and M. Virasoro, Spin glass theory and beyond: An Introduction to the Replica Method and Its Applications, Vol. 9 (World Scientific Publishing Co Inc, 1987).

[15] T. M. Cover and J. A. Thomas, Elements of information theory (John Wiley \& Sons, 2012).

[16] L. Kozachenko and N. N. Leonenko, Probl. Inf. Transm. 23, 9 (1987).

[17] B. Rennie and A. Dobson, J. Comb. Theory 7, 116 (1969).

[18] S. Dasgupta, in Foundations of computer science, 1999. 40th annual symposium on (IEEE, 1999) pp. 634-644.

[19] T. T. Cai, T. Liang, and H. H. Zhou, J. Multivariate Anal. 137, 161 (2015).

[20] D. Dheeru and E. Karra Taniskidou, "UCI machine learning repository," (2017).

[21] W. N. Street, W. H. Wolberg, and O. L. Mangasarian, in Biomedical Image Processing and Biomedical Visualization, Vol. 1905 (International Society for Optics and Photonics, 1993) pp. $861-871$.

[22] J. Friedman, T. Hastie, and R. Tibshirani, The elements of statistical learning, Vol. 1 (Springer, 2001).

[23] S. Jung, J. S. Marron, et al., The Annals of Statistics 37, 4104 (2009).

[24] N. G. De Bruijn, Asymptotic methods in analysis, Vol. 4 (Courier Corporation, 1970).

[25] B. Djokić, M. Miyakawa, S. Sekiguchi, I. Semba, and I. Stojmenović, Comput. J. 32, 281 (1989). 